\title{
What's in a name; Genetic structure in Solanum section Petota studied using population-genetic tools
}

\author{
Mirjam MJ Jacobs ${ }^{1,2,3}$, Marinus JM Smulders ${ }^{1}$, Ronald G van den Berg ${ }^{2,3}$, Ben Vosman ${ }^{1,3^{*}}$
}

\begin{abstract}
Background: The taxonomy and systematic relationships among species of Solanum section Petota are complicated and the section seems overclassified. Many of the presumed (sub)species from South America are very similar and they are able to exchange genetic material. We applied a population genetic approach to evaluate support for subgroups within this material, using AFLP data. Our approach is based on the following assumptions: (i) accessions that may exchange genetic material can be analyzed as if they are part of one gene pool, and (ii) genetic differentiation among species is expected to be higher than within species.

Results: A dataset of 566 South-American accessions (encompassing 89 species and subspecies) was analyzed in two steps. First, with the program STRUCTURE 2.2 in an 'unsupervised' procedure, individual accessions were assigned to inferred clusters based on genetic similarity. The results showed that the South American members of section Petota could be arranged in 16 clusters of various size and composition. Next, the accessions within the clusters were grouped by maximizing the partitioning of genetic diversity among subgroups (i.e., maximizing Fst values) for all available individuals of the accessions (2767 genotypes). This two-step approach produced an optimal partitioning into 44 groups.

Some of the species clustered as genetically distinct groups, either on their own, or combined with one or more other species. However, accessions of other species were distributed over more than one cluster, and did not form genetically distinct units.

Conclusions: We could not find any support for 43 species (almost half of our dataset). For 28 species some level of support could be found varying from good to weak. For 18 species no conclusions could be drawn as the number of accessions included in our dataset was too low. These molecular data should be combined with data from morphological surveys, with geographical distribution data, and with information from crossing experiments to identify natural units at the species level. However, the data do indicate which taxa or combinations of taxa are clearly supported by a distinct set of molecular marker data, leaving other taxa unsupported. Therefore, the approach taken provides a general method to evaluate the taxonomic system in any species complex for which molecular data are available.
\end{abstract}

\section{Background}

The taxonomy of wild potato species, belonging to section Petota of the genus Solanum, is known to be problematic [1-3]. Identification of many species is difficult and the systematic relationships among the wild potatoes are not clear. One of the causes for these

\footnotetext{
* Correspondence: ben.vosman@wur.nl

${ }^{1}$ Wageningen UR Plant Breeding, Wageningen University and Research Centre, Droevendaalsesteeg 1, 6708PB Wageningen, The Netherlands Full list of author information is available at the end of the article
}

difficulties is the ability of many species to hybridize easily [2]. Hawkes [1] hypothesized that approximately $12 \%$ of the 224 tuber-bearing Solanum species he recognized, had arisen from hybrid speciation. A quote from Correll [4] (page 404) may serve to illustrates the magnitude of the problem: "In fact, the difficulty one encounters in dealing with plants from northwest Argentina and southern Bolivia is such that one is tempted to consider, with very few exceptions the entire

\section{Biomed Central}


Tuberarium population to be one vast assemblage of hybrids" (section Tuberarium being roughly equivalent to the current section Petota).

Next to hybridization there is a large amount of phenotypic plasticity, i.e., plants look different in different environments [4-6]. Partly because of this, taxonomists have granted minor variants (sub)species status. As a consequence, species boundaries are based on morphological characters that are not expressed under all conditions. Hence, numerous species have been described, many of which are extremely similar to each other, and that is why Spooner and Salas [2] and van den Berg and Jacobs [3] concluded that the group of wild species belonging to Solanum section Petota is overclassified. An extreme example of overclassification within Solanum section Petota is the so-called brevicaule complex. Morphological results failed to distinguish the 30 species in the brevicaule complex [7]. Molecular data showed that the brevicaule complex is paraphyletic and that many taxa should probably be relegated to synonymy [8].

The systematic relationships among these species are also hard to determine. These have been expressed in an arrangement of 19 series, as designated by Hawkes [1] and others. Some of the series are difficult to keep apart while other series contain subgroups that could be considered a separate series [3]. To date, the series classification of Hawkes [1] and other authors has received no cladistic support [6]. Jacobs et al. [9] described the taxonomic structure present in Solanum section Petota. They focused on testing the validity of the series classification and on studying the taxonomic structure of the section based on AFLP data. They produced the largest dataset ever constructed for Solanum section Petota and analysed it both phenetically and phylogenetically. Although some of the branches in the resulting trees were supported by jackknife values above 69 , both phenetic and phylogenetic trees also display a large polytomy containing many taxa.

In the present study, we focus on the status of the recognized species in section Petota, in order to evaluate possible overclassification, misclassification and hybridization. The number of species in the Solanum section Petota has already been reduced somewhat due to the application of molecular techniques. While Hawkes [1] still recognized 227 tuber-bearing species (of which 7 were cultivated) and 9 non tuber-bearing species within section Petota, Spooner and Hijmans [5] recognized only 203 tuber-bearing species, including 7 cultivated species. Spooner and Salas [2] reduced the number further to 189 species (including only 1 cultivated species). Phylogenetic and phenetic analysis of previous studies, reviewed in van den Berg and Jacobs [3] and Jacobs et al. [9] revealed that accessions from many wild Solanum species, especially the species of the South
American series Tuberosa, Megistacroloba, and Yungasensia, are closely related. This is consistent with the observations that they freely exchange genes and produce hybrids under artificial conditions. Because of this, we chose as the starting point of our analysis the AFLP data used by Jacobs et al. [9] to consider the individual plants as belonging to one gene pool, rather than to separate taxa, and to employ a population genetics approach to detect the genetic structure of these AFLP data for the group of South American representatives of Solanum section Petota.

To test which accessions may belong to one or more species groups we used a Bayesian population clustering approach implemented in the program STRUCTURE $2.2[10,11]$. STRUCTURE clusters individuals without using a-priori information about their identity. The primary assumptions of the model used in STRUCTURE are Hardy-Weinberg equilibrium (HWE) within populations and linkage equilibrium among loci, and the program attempts to find population groupings that are not in disequilibrium [11]. Both assumptions may not always be valid when taking a more or less random set of accessions collected over a larger area as representing a species, but disequilibrium will always be smaller within a species than between species. The program has been successfully used in a large variety of population genetic studies, for example in the research of genetic structure in the human population [12], in the phylogeography of the sand-dune shrub America pungens [13], for distinguishing chicken breeds [14], and to detect hybrids between cultivated and wild apple $[15,16]$. Recently, STRUCTURE was also used in studies on phylogenetic relationships among birch species [17], on species delimitation in a recent species radiation in turtles [18] and in the Mexican jay [19], and produced part of the evidence for a separate species status of the Galapagos sea lion [20].

Accessions within one species are expected to share more alleles with each other than with accessions from other species. As a result, genetic differentiation among species is expected to be higher than within species. Consequently, if we subdivide an unstructured set of accessions according to their species labels, the fraction of the genetic variation present among, rather than within, groups will be higher if those species labels correctly identify the accessions. If the species labels are incorrect then combining accessions with incorrect species labels into new groups will increase the fraction genetic variation among the groups.

Thus, the genetic differentiation among alternative groupings as expressed in Fst values will allow us to further subdivide the groups resulting from the STRUCTURE analysis, and to distinguish genetically separate species from species that should be grouped together. 
This approach of species delimitation resembles somewhat the view of Shaffer and Thompson [18] that follows Mayden [21] and de Queiroz [22,23], in that they consider species as segments of evolutionary lineages. In this view, species delimitation comes down to identification of metapopulation-like lineages. The metapopulation lineage species definition leads to operational species delimitation approaches that recognize sets of populations that freely exchange genes in nature but have no or very restricted gene exchange with other sets of populations [18]. In this paper we describe how this approach works out for Solanum section Petota.

\section{Methods}

\section{Plant Material}

We used the plant material from the genus Solanum section Petota as described in Jacobs et al. [9], which consists of 4929 genotypes representing 916 accessions. From each accession a representative genotype was chosen [9]. A subset (out of the 916) consisting of 566 plants (one plant per accession) was made, representing the 89 species/subspecies from South America that appeared in the large polytomy of the trees presented by Jacobs et al. [9], plus the accessions that do not belong to the species groups with high jackknife or bootstrap support (viz. excluding the following supported groups: Acaulia group, Mexican diploid group, diploid Piurana group, tetraploid Piurana group, polyploid Conicibaccata group, diploid Conicibaccata group, Circaeifolia group, Longipedicellata group, and Iopetala group). Information on the accession numbers and geographic origin of these 566 samples can be found in Additional file 1 . The nomenclature of the plant material follows that of Jacobs et al. [9]. This means that in some cases we have retained the original labels, even when taxonomic references suggested a change of the species name. However, a number of obvious mistakes (due to mislabeling) that became clear after preliminary AFLP analyses have been corrected after morphological examination.

\section{AFLP}

The protocol of Vos et al. [24] was used to generate AFLP fragments. The plant material was fingerprinted with two EcoRI/MseI AFLP primer combinations: E32/ M49 and E35/M48. These primer combinations gave 91 and 131 polymorphic bands, respectively. The AFLP analysis was done on a MegaBACE 2.1 by Keygene N.V. Bands were scored as dominant markers, using the Keygene proprietary software.

\section{Data analysis \\ Bayesian clustering}

The 566 South-American accessions were analyzed with STRUCTURE $2.2[10,11]$ in an 'unsupervised' procedure according to Rosenberg [25] based on genetic similarities only. We used the approach of coding the dominant markers as described by Falush et al [26]. The dominant AFLP data were entered by coding both alleles as ' 1 ' when the AFLP band was present and both as '0' when the band was absent. We specified ' 0 ' as the recessive allele for all the AFLP data. This enables the simultaneous analysis of accessions with different levels of ploidy like described by Schenk et al. [17]. Evanno et al. [27] showed that results of AFLPs with STRUCTURE can be as accurate as those of microsatellites. Estimates for the log likelihood were obtained using the admixture model and the assumption that the allele frequencies are correlated. The log likelihood estimates were obtained for 10 replicate runs at each $\mathrm{K}$ ranging from $\mathrm{K}$ $=1$ to $\mathrm{K}=30$. For each run, we used a burn-in of 25,000 cycles and a data run of 100,000 cycles.

To test whether STRUCTURE was suitable for analyzing the Solanum AFLP data, a pilot analysis was carried out on the condensed dataset of 916 individuals. Almost all species groups as defined by Jacobs et al. [9] and smaller supported branches in the NJ tree have their own cluster at $\mathrm{K}=18$ or higher (results not shown), which confirms that STRUCTURE can be used for the AFLP dataset.

Partitioning of genetic variation within and among groups It is unrealistic to assume that one STRUCTURE analysis could separate all species. Some of the 566 accessions may be from a genetically homogeneous species that occupies a small area, while others may be from a genetically highly variable species that occupies a large area. Some species were represented by many accessions, others by only a few. Therefore, while increasing the number of clusters (K) in the STRUCTURE analyses, accessions of certain species may already start to be assigned to different clusters before accessions of other species would be separated from each other. When large datasets are analyzed convergence problems for the Gibbs sampler algorithm used in STRUCTURE software may occur $[12,28]$. Therefore we decided do a nested analysis.

The second level (nested) analyses could be done again by STRUCTURE for each group separately, as e.g. Jing et al. [29] did in Pisum. The advantage is that an a priori grouping is made and accessions formerly classified under the same name may end up in different groups. An alternative option was to optimize the grouping of accessions by maximizing the Fst among the species or among combinations of species. This has two important advantages: (1) all plants within an accession can be included in this computationally simple analysis, and (2) even if several rounds of grouping are performed, it is still much faster than optimizing and performing a STRUCTURE analysis on each of the 16 
clusters. A disadvantage is that accessions of the same name remain together, which may mean that in theory the best solution is less optimal than obtained with the nested STRUCTURE approach.

As a pilot experiment, we performed a nested STRUCTURE analysis on a few clusters and compared the results to an Fst analysis of the same clusters. The results were compared by calculating the Fst among groups for the nested STRUCTURE analysis and for the optimized Fst approach. The optimized Fst approach always resulted in a higher value for the Fst among the groups within the cluster (not shown). We therefore decided to continue with the Fst analysis. This combination is a novel approach.

The partitioning of genetic variation (Fst) among STRUCTURE clusters or among new groups within a cluster was computed using AFLP-SURV 1.0 [30]. The allelic frequencies at AFLP loci were calculated from the observed frequencies of fragments, using the Bayesian approach [31] (assuming diploid species and HardyWeinberg equilibrium) using all 2767 available genotypes for the 566 accessions (when available 5 plants per accession). We assumed a uniform prior distribution of allelic frequencies. Significance of the Fst values was tested by 1000 permutations. The confidence limits obtained were used to determine the significance of differences between the separate estimates.

\section{Grouping within clusters by maximizing Fst}

Within each of the 16 STRUCTURE clusters we calculated Fst based on the species present using AFLP-Surv. Subsequently, combinations of accessions with different species labels were made and the overall Fst value and pairwise Fst values between the groups within a cluster were computed. We performed several rounds of grouping. Each time the accessions of those species or groups that showed a pairwise Fst of less than the observed overall Fst of the groups within the cluster were combined. This process was repeated, merging species and species groups, until further merging of groups did not increase the overall Fst value significantly.

\section{Results}

\section{Clustering of the 566 South-American accessions into 16} clusters

The 566 South-American accessions were analyzed using STRUCTURE, testing various numbers of groups, from $K=1$ to $K=30$. Figure 1 shows the average posterior probability $\operatorname{Ln}(\mathrm{P}(\mathrm{D}))$ for 10 runs as a function of

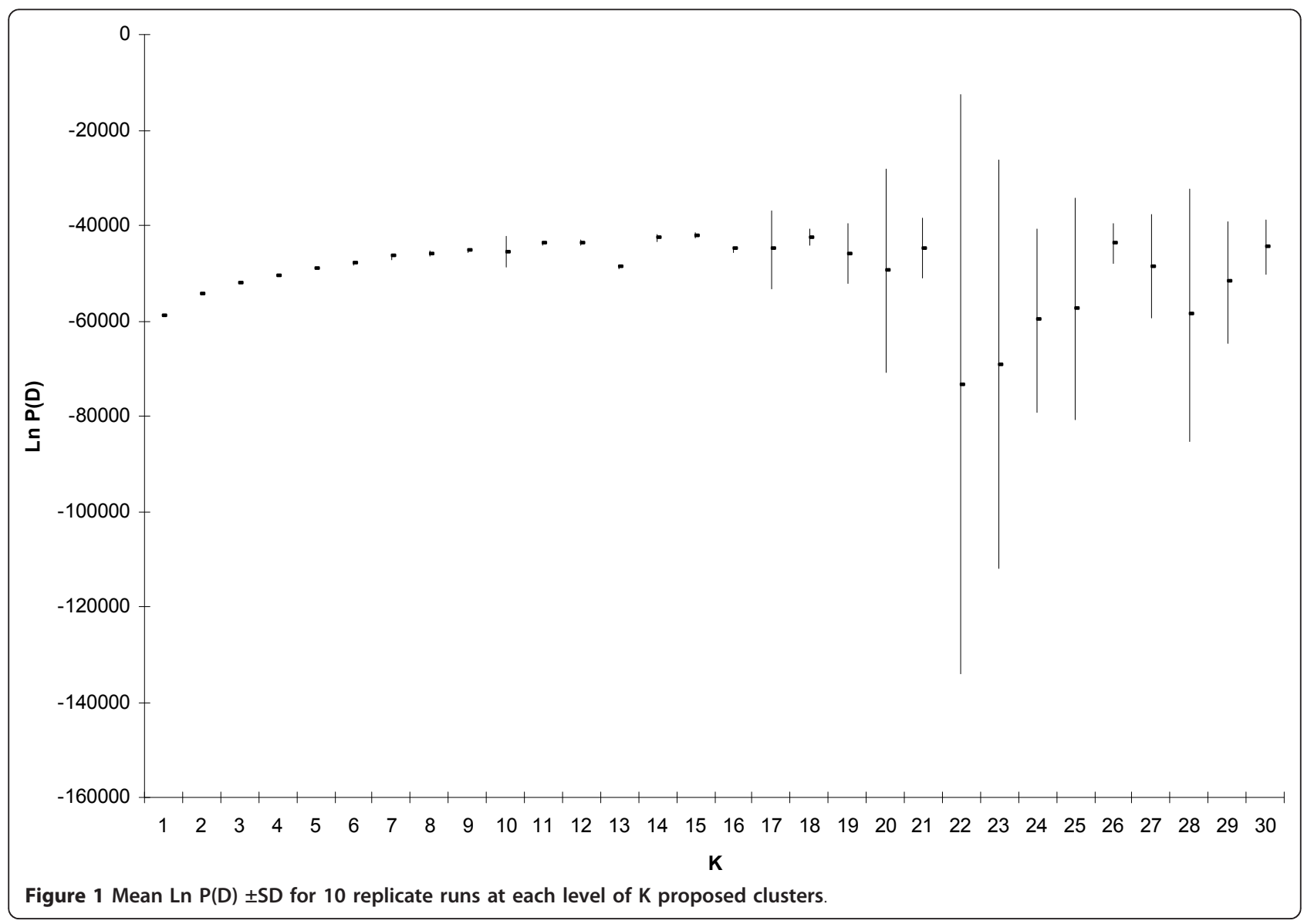


$\mathrm{K}$. The posterior probability increases until around $\mathrm{K}=$ 16 , after which it reaches a plateau. From $\mathrm{K}=18$ onwards the posterior probability became increasingly variable among runs, and the clustering of accessions became unstable between replicate runs. In contrast, at $\mathrm{K}=16$ the clustering results were stable and most clusters had the same composition in all 10 replicate runs. We therefore took $\mathrm{K}=16(\operatorname{Ln} \mathrm{P}(\mathrm{D})=-41181.7)$ as the optimal K.

The estimated population structure of one run at $\mathrm{K}=$ 16 is shown in Figure 2. Each individual accession is presented by a thin vertical line, and this line shows colored segments that represent the relative percentage of membership to the $\mathrm{K}$ clusters (the underlying data can be found in Additional file 1). The accessions labeled as S. okadae, S. raphanifolium, S. verrucosum, and $S$. macropilosum occupy exclusively one cluster, while many other accessions are found to share a cluster with accessions from one or more other species, for instance $S$. huancabambense with $S$. sogarandinum. Many accessions labeled with the same species name are distributed over two clusters, e.g. the accessions of S. maglia, S. gourlayi, S. tarijense. Finally, there is a number of species whose accessions show membership to more than two clusters. Additional file 1 provides the detailed results on the composition of the clusters and the percentage of membership per individual accession for these clusters, in the run with the highest probability. Most clusters defined by STRUCTURE for $\mathrm{K}=16$ are the same in all 10 runs. The main exception is cluster 3 , which was found in only 3 out of 10 runs as a separate unit. In the other 7 runs its accessions were combined with those of cluster 4 .

The partitioning of genetic variation among the clusters (Fst) in the 16 cluster arrangement represented 31\% of the genetic variation (Table 1). For comparison, we also calculated that the 89 pre-existing taxa explained $29 \%$ of the existing genetic variation. A subdivision in

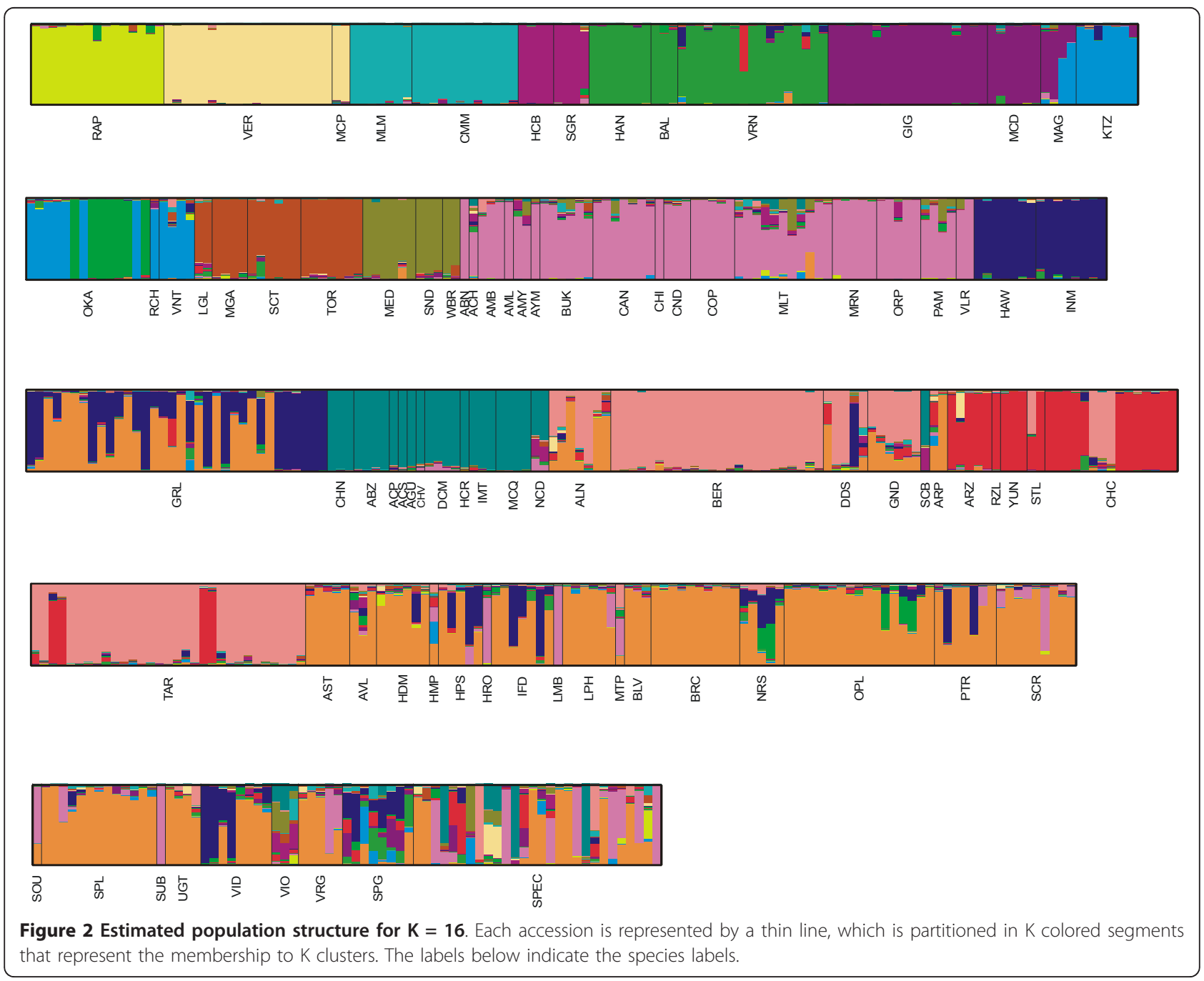


Table 1 Genetic differentiation in complete dataset

\begin{tabular}{|c|c|c|c|c|c|c|}
\hline & $\mathrm{N}^{*}$ & $\mathrm{Ht}$ & $\mathrm{Hw}$ & $\mathrm{Hb}$ & Fst & $p$-value \\
\hline among the accessions & 538 & 0.3256 & 0.2783 & 0.0473 & 0.1453 & $<0.001$ \\
\hline $\begin{array}{l}\text { among the old species } \\
\text { labels }\end{array}$ & 89 & 0.2632 & 0.1855 & 0.0777 & 0.2953 & $<0.001$ \\
\hline $\begin{array}{l}\text { among the clusters } \\
\text { at } k=16\end{array}$ & 16 & 0.2077 & 0.1430 & 0.0647 & 0.3124 & $<0.001$ \\
\hline $\begin{array}{l}\text { among the clusters } \\
\text { at } k=10\end{array}$ & 10 & 0.2023 & 0.1475 & 0.0548 & 0.2733 & $<0.001$ \\
\hline $\begin{array}{l}\text { among new subgroups } \\
\text { (see table 2) }\end{array}$ & 44 & 0.2438 & 0.1594 & 0.0844 & 0.3464 & $<0.001$ \\
\hline
\end{tabular}

*28 accessions labeled unknown species were excluded in this analysis. $\mathrm{n}=$ number of sampled accessions/groups; $\mathrm{Ht}=$ total diversity; $\mathrm{Hw}=$ within population diversity; $\mathrm{Hb}=$ between population diversity; Fst = Wright's fixation index, differentiation among populations.

10 groups (one run of a suboptimal STRUCTURE analysis at $\mathrm{K}=10$ ) already explained $27 \%$. The 566 individual accession arrangement showed the lowest value of Fst, as only $15 \%$ of the genetic variation is present among accessions. All Fst values were significantly different from each other.

The level of genetic differentiation among the accessions was lower within the clusters than among the clusters (Table 2). The lowest values are for cluster 1, 6 and 15 , which mainly or exclusively consist of accessions with only one species label, e.g. cluster 15, which contains only $S$. okadae accessions, has an Fst of 0.0029.

Genetic differentiation among species within clusters that contain accessions from two species ranged from $9.8 \%$ in cluster 4 to $27.8 \%$ in cluster 7 . In cluster 4 , cluster 10 , and cluster 12 the species arrangement only added a small part to the genetic differentiation, relative to the value for all accessions separately.

\section{Further subdivision of the 16 clusters}

As the contribution to the partitioning of genetic variation could differ for the various species within a cluster, we performed several rounds of grouping on all 2767 individuals available for these accessions. Each time the accessions of those species that showed a pairwise Fst of less than the observed overall Fst of the groups within the cluster were combined into one group, so that in the next round the number of groups was lower. The process was repeated, merging species and species groups, until further merging did not increase the Fst value. Table 2 lists the Fst value of the optimal number of groups, along with those of the value obtained with one group more or less, and the group structure of the optimal configuration is reported. In most of the clusters one or two merging steps were sufficient to reach a maximum Fst, but in cluster 7, 12, and 14, three cycles were needed, while in cluster 10 and 16 the process took four cycles. In some clusters the highest overall Fst was reached when most of the species labels were merged together; this was the case in cluster 10, 14 and 16. In other clusters the optimal Fst was reached at an arrangement that only merged a few of the species in the cluster, while other species remained separate. This was the case in cluster 3, 4 and 13. In cluster 8 no new arrangement yielded a higher Fst. Overall, the 566 accessions were grouped into 44 genetically distinct groups.

The assignment of the 566 accessions into 44 genetically distinct groups was then used to infer the support for the 89 species into which these accessions had been classified. The results are presented according to taxonomical classification in Table 3 , and will be discussed below. For those species (18) that were represented by only one accessions in this study, no conclusion could be drawn. For 43 species there was no evidence, for 20 there was weak evidence and for 8 there was good evidence.

\section{Discussion}

Many described species in section Petota are very similar to each other and are able to cross, suggesting that this section is overclassified. We have tested this for the large group of South American species of the section Petota, using a population genetic approach that would allow us to identify any structure among this material, if present. The results obtained from the analysis of 566 South-American Solanum section Petota accessions with STRUCTURE showed an optimal overall subdivision of these accessions in 16 clusters. By maximizing the partitioning of genetic variation among groups (Fst) we obtained support for additional groups within these clusters, up to a total of 44 units (or 48 units including the unknown species accessions) (Table 2). This does not automatically mean that 44 is the correct number of species as genetic differentiation would be expected among separate species but it can also be found among populations within a species (see below). Nevertheless, the Fst values of the various species arrangements in Table 1 offer a clear indication of overclassification: Fst increases from 0.145 (the 566 accessions) to 0.273 (10 clusters) and to 0.312 (16 clusters). The highest value is obtained after the nested analysis, when 44 groups explain $35 \%$ of the genetic variation (the remainder being present within species). The Fst value of the 89 species arrangement $(0.2953)$ is even lower than that of the 16 clusters $(0.312)$, indicating that the current species arrangement is 'over the top' but still does explain a considerable part of the genetic variation within the dataset.

\section{Misclassification and overclassification}

If not all accessions of a species are in one cluster but one or a few are present in different clusters, this may 
Table 2 Genetic differentiation within the 16 clusters

\begin{tabular}{|c|c|c|c|c|c|c|c|c|}
\hline Cluster & species included in cluster & sampling units & $\mathrm{n}$ & $\mathrm{Ht}$ & $\mathrm{Hw}$ & $\mathrm{Hb}$ & Fst & $\begin{array}{l}\text { species included in the clusters/new } \\
\text { arrangements }\end{array}$ \\
\hline 1 & rap (15) & $\begin{array}{l}\text { among } \\
\text { accessions }\end{array}$ & 15 & 0.2679 & 0.2776 & -0.0098 & -0.0365 & \\
\hline \multirow[t]{4}{*}{2} & ver (19), mcp (2) spec 262, spec 287 & $\begin{array}{l}\text { among } \\
\text { accessions }\end{array}$ & 23 & 0.2515 & 0.2448 & 0.0068 & 0.0272 & \\
\hline & & among species & 4 & 0.2765 & 0.2063 & 0.0702 & 0.2377 & \\
\hline & & $\begin{array}{l}\text { among new } \\
\text { groups }\end{array}$ & 3 & 0.2445 & 0.1551 & 0.0894 & 0.3598 & \\
\hline & & $\begin{array}{l}\text { among new } \\
\text { groups }\end{array}$ & 2 & 0.2859 & 0.1471 & 0.1388 & 0.4675 & (ver, mcp) (spec 262, spec287) \\
\hline \multirow[t]{4}{*}{3} & $\begin{array}{l}\text { ktz(7), mag (2), oka (8), rch (1), spg (1), } \\
\text { vnt (4) }\end{array}$ & $\begin{array}{l}\text { among } \\
\text { accessions }\end{array}$ & 22 & 0.2875 & 0.2704 & 0.0171 & 0.0592 & \\
\hline & & among species & 6 & 0.2438 & 0.2113 & 0.0325 & 0.1285 & \\
\hline & & $\begin{array}{l}\text { among new } \\
\text { groups }\end{array}$ & 3 & 0.2 & 0.1527 & 0.0473 & 0.2319 & (ktz, mag, rch) (oka, vnt) spg \\
\hline & & $\begin{array}{l}\text { among new } \\
\text { groups }\end{array}$ & 2 & 0.1686 & 0.1305 & 0.0381 & 0.2231 & \\
\hline \multirow[t]{4}{*}{4} & gig(18), mag (2), mcd (6) spg (1) & $\begin{array}{l}\text { among } \\
\text { accessions }\end{array}$ & 27 & 0.2556 & 0.2457 & 0.0098 & 0.0384 & \\
\hline & & among species & 4 & 0.1973 & 0.1777 & 0.0195 & 0.0984 & \\
\hline & & $\begin{array}{l}\text { among new } \\
\text { groups }\end{array}$ & 3 & 0.213 & 0.1898 & 0.0231 & 0.1059 & (gig, mcd) mag spg \\
\hline & & $\begin{array}{l}\text { among new } \\
\text { groups }\end{array}$ & 2 & 0.3341 & 0.3064 & 0.0277 & 0.0639 & \\
\hline \multirow[t]{4}{*}{5} & med (6), snd (3), wbr (2) vio (1) & $\begin{array}{l}\text { among } \\
\text { accessions }\end{array}$ & 13 & 0.303 & 0.2864 & 0.0166 & 0.0548 & \\
\hline & & among species & 4 & 0.2594 & 0.2069 & 0.0525 & 0.2023 & \\
\hline & & $\begin{array}{l}\text { among new } \\
\text { groups }\end{array}$ & 3 & 0.2626 & 0.1949 & 0.0678 & 0.2559 & \\
\hline & & $\begin{array}{l}\text { among new } \\
\text { groups }\end{array}$ & 2 & 0.2926 & 0.2055 & 0.0871 & 0.2893 & (snd, wbr, med) vio \\
\hline \multirow[t]{2}{*}{6} & $\mathrm{cmm}(12), \mathrm{m} / \mathrm{m}(7)$ & $\begin{array}{l}\text { among } \\
\text { accessions }\end{array}$ & 19 & 0.3357 & 0.3535 & -0.0177 & -0.0528 & \\
\hline & & among species & 2 & 0.1763 & 0.1395 & 0.0368 & 0.2084 & $\mathrm{cmm} \mathrm{m} / \mathrm{m}$ \\
\hline \multirow[t]{6}{*}{7} & $\begin{array}{l}\text { abz (4), acp (1), acs (1), agu (1), chn (3), } \\
\text { chv (1), dcm (4), hcr (1), imt (3), mcq } \\
\text { (4), ncd (2), scb (1) vio (1) spec 205, } \\
\text { spec 310, spec } 6\end{array}$ & $\begin{array}{l}\text { among } \\
\text { accessions }\end{array}$ & 30 & 0.3016 & 0.2449 & 0.0567 & 0.1878 & \\
\hline & & among species & 16 & 0.2754 & 0.1988 & 0.0766 & 0.2777 & \\
\hline & & $\begin{array}{l}\text { among new } \\
\text { groups }\end{array}$ & 7 & 0.2448 & 0.143 & 0.1019 & 0.4175 & \\
\hline & & $\begin{array}{l}\text { among new } \\
\text { groups }\end{array}$ & 6 & 0.245 & 0.1405 & 0.1045 & 0.4276 & \\
\hline & & $\begin{array}{l}\text { among new } \\
\text { groups }\end{array}$ & 5 & 0.2513 & 0.1419 & 0.1094 & 0.4357 & $\begin{array}{l}\text { (acs, agu, hcr, acp, scb, chn, mcq, } \\
\text { imt, spec 205, spec 310, spec 6) (dcm, } \\
\text { chv) ncd, abz, vio }\end{array}$ \\
\hline & & $\begin{array}{l}\text { among new } \\
\text { groups }\end{array}$ & 4 & 0.25 & 0.1418 & 0.1082 & 0.4333 & \\
\hline \multirow[t]{3}{*}{8} & hcb(4), sgr (3), vio (1) & $\begin{array}{l}\text { among } \\
\text { accessions }\end{array}$ & 9 & 0.2813 & 0.2319 & 0.0494 & 0.1758 & \\
\hline & & among species & 3 & 0.2774 & 0.1919 & 0.0856 & 0.3043 & hcb, sgr, vio \\
\hline & & $\begin{array}{l}\text { among new } \\
\text { groups }\end{array}$ & 2 & 0.3053 & 0.2128 & 0.0925 & 0.2948 & \\
\hline
\end{tabular}


Table 2 Genetic differentiation within the 16 clusters (Continued)

\begin{tabular}{|c|c|c|c|c|c|c|c|c|}
\hline \multirow[t]{4}{*}{9} & bal(3), han (7), vrn (16), spg (1) & $\begin{array}{l}\text { among } \\
\text { accessions }\end{array}$ & 27 & 0.2659 & 0.2434 & 0.0226 & 0.0847 & \\
\hline & & among species & 4 & 0.2092 & 0.1687 & 0.0405 & 0.1904 & \\
\hline & & $\begin{array}{l}\text { among new } \\
\text { groups }\end{array}$ & 3 & 0.2133 & 0.1665 & 0.0468 & 0.2114 & \\
\hline & & $\begin{array}{l}\text { among new } \\
\text { groups }\end{array}$ & 2 & 0.1662 & 0.1251 & 0.0411 & 0.2459 & (bal, vrn, spg) han \\
\hline \multirow[t]{7}{*}{10} & $\begin{array}{l}\text { abn (1), ach(1), amb (3), aml(1), amy } \\
(2) \text {, aym (1), buk (6), can (7), chi (1), } \\
\text { cnd (3), cop (5), hro (1), Imb (1), mlt } \\
(11) \text {, mrn (5), orp (5), pam (4), sou (1), } \\
\text { scr (1), sub (1) vlr (2) vrg (1) spec 998, } \\
\text { spec 184, spec 292, spec 533, spec } \\
\text { 726, spec 796, spec 394, spec } 933\end{array}$ & $\begin{array}{l}\text { among } \\
\text { accessions }\end{array}$ & 72 & 0.3095 & 0.2881 & 0.0213 & 0.069 & \\
\hline & & among species & 31 & 0.2828 & 0.2529 & 0.03 & 0.1058 & \\
\hline & & $\begin{array}{l}\text { among new } \\
\text { groups }\end{array}$ & 16 & 0.2598 & 0.2171 & 0.0427 & 0.1645 & \\
\hline & & $\begin{array}{l}\text { among new } \\
\text { groups }\end{array}$ & 7 & 0.2574 & 0.2045 & 0.0529 & 0.2066 & \\
\hline & & $\begin{array}{l}\text { among new } \\
\text { groups }\end{array}$ & 5 & 0.2796 & 0.2195 & 0.0601 & 0.2143 & \\
\hline & & $\begin{array}{l}\text { among new } \\
\text { groups }\end{array}$ & 4 & 0.2996 & 0.2336 & 0.066 & 0.2194 & $\begin{array}{l}\text { (abn, ach, amb, aml, amy, aym, buk, } \\
\text { can, chi, cnd, cop, hro, Imb, mlt, mrn } \\
\text { orp, pam, sou, scr, sub, vlr, vrg, spec } \\
\text { 998, spec 184, mtp, spec 292, spec } \\
\text { 533, spec 726, spec 796) ach, } \\
\text { spec933, spec394 }\end{array}$ \\
\hline & & $\begin{array}{l}\text { among new } \\
\text { groups }\end{array}$ & 3 & 0.2748 & 0.2142 & 0.0606 & 0.2189 & \\
\hline \multirow[t]{4}{*}{11} & Igl (2), mga (4), sct (6), tor (7) & $\begin{array}{l}\text { among } \\
\text { accessions }\end{array}$ & 19 & 0.3231 & 0.31 & 0.0131 & 0.0401 & \\
\hline & & among species & 4 & 0.2285 & 0.1885 & 0.04 & 0.1743 & \\
\hline & & $\begin{array}{l}\text { among new } \\
\text { groups }\end{array}$ & 3 & 0.2421 & 0.1968 & 0.0453 & 0.1858 & (mga, tor) $l g l, s c t$ \\
\hline & & $\begin{array}{l}\text { among new } \\
\text { groups }\end{array}$ & 2 & 0.2269 & 0.1877 & 0.0391 & 0.1704 & \\
\hline \multirow[t]{6}{*}{12} & $\begin{array}{l}\operatorname{arz}(5), \operatorname{chc}(12), \operatorname{rzl}(1), \operatorname{st}(1), \text { yun (3), } \\
\operatorname{tar}(4), \operatorname{vrn}(1) \text { spec } 210, \text { spec211, } \\
\text { spec329, grl(1) }\end{array}$ & $\begin{array}{l}\text { among } \\
\text { accessions }\end{array}$ & 31 & 0.2927 & 0.2716 & 0.0211 & 0.072 & \\
\hline & & among species & 11 & 0.2784 & 0.2506 & 0.0278 & 0.0991 & \\
\hline & & $\begin{array}{l}\text { among new } \\
\text { groups }\end{array}$ & 6 & 0.2236 & 0.1867 & 0.0369 & 0.1646 & \\
\hline & & $\begin{array}{l}\text { among new } \\
\text { groups }\end{array}$ & 4 & 0.22 & 0.178 & 0.042 & 0.1886 & \\
\hline & & $\begin{array}{l}\text { among new } \\
\text { groups }\end{array}$ & 3 & 0.2317 & 0.1853 & 0.0465 & 0.1949 & $\begin{array}{l}\text { (chc, rzl, stl, grl, spec210, spec211, } \\
\text { spec329, yun, arz) vrn, arz }\end{array}$ \\
\hline & & $\begin{array}{l}\text { among new } \\
\text { groups }\end{array}$ & 2 & 0.1772 & 0.1445 & 0.0326 & 0.1831 & \\
\hline \multirow[t]{4}{*}{13} & $\begin{array}{l}\text { dds (1), grl (15), hps (3), ifd (2), inm (8), } \\
\text { ptr (3), vid (3), nrs (2), spg (3), haw (7) }\end{array}$ & $\begin{array}{l}\text { among } \\
\text { accessions }\end{array}$ & 47 & 0.3045 & 0.2885 & 0.016 & 0.0526 & \\
\hline & & among species & 10 & 0.2252 & 0.18 & 0.0452 & 0.2006 & \\
\hline & & $\begin{array}{l}\text { among new } \\
\text { groups }\end{array}$ & 5 & 0.2135 & 0.1582 & 0.0553 & 0.259 & $\begin{array}{l}\text { (grl, dds, hps, ifd, inm, ptr) vid, haw, } \\
\text { spg, nrs }\end{array}$ \\
\hline & & $\begin{array}{l}\text { among new } \\
\text { groups }\end{array}$ & 4 & 0.2066 & 0.1545 & 0.0521 & 0.2522 & \\
\hline 14 & $\begin{array}{l}\operatorname{chc}(3), \operatorname{dds}((3), \operatorname{tar}(27), \operatorname{aln}(4), \operatorname{ber}(23), \\
\text { gnd(5), stl(1), spec 255, spec } 601\end{array}$ & $\begin{array}{l}\text { among } \\
\text { accessions }\end{array}$ & 69 & 0.2671 & 0.2518 & 0.0153 & 0.0573 & \\
\hline
\end{tabular}


Table 2 Genetic differentiation within the 16 clusters (Continued)

\begin{tabular}{|c|c|c|c|c|c|c|c|}
\hline & among species & 9 & 0.2092 & 0.1823 & 0.027 & 0.1276 & \\
\hline & $\begin{array}{l}\text { among new } \\
\text { groups }\end{array}$ & 6 & 0.1726 & 0.148 & 0.0246 & 0.1417 & \\
\hline & $\begin{array}{l}\text { among new } \\
\text { groups }\end{array}$ & 5 & 0.1766 & 0.1502 & 0.0264 & 0.1479 & \\
\hline & $\begin{array}{l}\text { among new } \\
\text { groups }\end{array}$ & 4 & 0.157 & 0.1327 & 0.0244 & 0.1549 & $\begin{array}{l}\text { (stl, spec } 255, \text { spec } 601, \text { yun, chc, dds, } \\
\text { tar) aln ber gnd }\end{array}$ \\
\hline & $\begin{array}{l}\text { among new } \\
\text { groups }\end{array}$ & 3 & 0.1616 & 0.1383 & 0.0233 & 0.1439 & \\
\hline 15 oka (7) & $\begin{array}{l}\text { among } \\
\text { accessions }\end{array}$ & 7 & 0.261 & 0.2602 & 0.0007 & 0.0029 & \\
\hline \multirow[t]{7}{*}{$\begin{array}{l}16 \text { grl (18), hps(2), aln(3), arp(2), dds(1), } \\
\text { hmp(1) ptr(5), scr(8), Iph(6), ugt(4), vrg } \\
\text { (4), opl(17), ifd(5), vid(5), gnd(1), vrn(1), } \\
\text { nrs(2), spg(1), spl(13), brc(10), ast(5), blv } \\
\text { (3), hdm(6), avl(3) spec352, spec43, } \\
\text { spec123, spec165, spec891, spec381, } \\
\text { spec649 }\end{array}$} & $\begin{array}{l}\text { among } \\
\text { accessions }\end{array}$ & 134 & 0.3169 & 0.302 & 0.015 & 0.0473 & \\
\hline & among species & 31 & 0.2456 & 0.215 & 0.0306 & 0.1245 & \\
\hline & $\begin{array}{l}\text { among new } \\
\text { groups }\end{array}$ & 14 & 0.217 & 0.1823 & 0.0347 & 0.1597 & \\
\hline & $\begin{array}{l}\text { among new } \\
\text { groups }\end{array}$ & 7 & 0.214 & 0.1742 & 0.0399 & 0.1855 & \\
\hline & $\begin{array}{l}\text { among new } \\
\text { groups }\end{array}$ & 6 & 0.2166 & 0.1749 & 0.0418 & 0.1913 & \\
\hline & $\begin{array}{l}\text { among new } \\
\text { groups }\end{array}$ & 5 & 0.224 & 0.1799 & 0.0442 & 0.1952 & $\begin{array}{l}\text { (grl, hps, aln, arp, dds, hmp ptr, scr, } \\
\text { lph, ugt, vrg, opl, ifd, vid, gnd, vrn, } \\
\text { nrs, spg, spl, brc, spec416, spec352, } \\
\text { spec43, spec123, spec165, spec891, } \\
\text { spec381), (ast, blv), hdm, avl, spec649 }\end{array}$ \\
\hline & $\begin{array}{l}\text { among new } \\
\text { groups }\end{array}$ & 4 & 0.2372 & 0.1932 & 0.0439 & 0.1831 & \\
\hline
\end{tabular}

$\mathrm{n}=$ number of sampled accessions; $\mathrm{Ht}=$ total diversity; $\mathrm{Hw}=$ within population diversity; $\mathrm{Hb}=$ between population diversity; Fst = Wright's fixation index, differentiation among populations. The explanation of the species code can be found in table 3 .

indicate misclassification. Occurrence of different species labels intermingled within one cluster points at overclassification. From both situations we see examples in our dataset and these may have consequences for the (sub) species status of the present taxa.

\section{Status of subspecies}

In nearly all cases there was no support for maintaining taxa at the subspecies level. This is the case for the subspecies within the species $S$. microdontum, S. vernei, $S$. boliviense and S. megistacrolobum. Only one of the recognized subspecies was supported in our analysis: $S$. commersonii subsp. malmeanum could be differentiated genetically from $S$. commersonii subsp. commersonii (Table 3).

Some of these (sub) species have been extensively studied previously, using morphology. The subspecies S. microdontum subsp. gigantophyllum was already considered to be a synonym of S. microdontum [32] and should not be recognized, as this is a clear case of overclassification. Giannattasio and Spooner studied the boundaries between $S$. megistacrolobum subsp. megistacrolobum and S. megistacrolobum subsp. toralapanum using morphological data [33] and with molecular markers [34]. Based on their analysis they suggested to preserve $S$. megistacrolobum subsp. toralapanum as a distinct subspecies while our analysis does not find support for this. Spooner et al. [35] studied the relationships of S. boliviense and S. astleyi using RAPDs and concluded that $S$. astleyi should be reduced to a subspecies of S. boliviense. Our data do not provide support for a subspecies level in S. boliviense.

\section{Some species are supported}

The following species are supported as genetically distinct units: S. raphanifolium, S. verrucosum (with $S$. macropilosum as synonym), S. microdontum, S. commersonii, S. okadae (only the seven accessions in cluster 15), S. huancabambense, and S. sogarandinum. The seven S. okadae accessions that appear in cluster 3 together with $S$. venturii accessions turned out to be mislabeled and have been corrected as being $S$. venturii accessions 
Table 3 Information on species labels and accessions used in the analysis and suggestions for species status

\begin{tabular}{|c|c|c|c|c|c|c|c|}
\hline $\begin{array}{l}\text { series } \\
\text { according to } \\
\text { Hawkes (1990) }\end{array}$ & species & $\begin{array}{l}\text { species } \\
\text { abbreviation }\end{array}$ & accessions code & $\begin{array}{l}\text { source codes } \\
\text { (genebank) }\end{array}$ & $\begin{array}{l}\text { (total } \mathrm{nr} \text {. } \\
\text { of } \\
\text { accessions } \\
\text { in } 566 \\
\text { dataset) }\end{array}$ & taxonomic remarks & $\begin{array}{l}\text { evidence for } \\
\text { species } \\
\text { status } \\
\text { according to } \\
\text { the authors }\end{array}$ \\
\hline Tuberosa II & $\begin{array}{l}\text { S. abancayense } \\
\text { Ochoa }\end{array}$ & $a b n$ & 423 & CGN 18357 & 1 & $\begin{array}{l}\text { synonym of S. } \\
\text { bukasovii (Ochoa, 1999) }\end{array}$ & $\begin{array}{l}\text { not enough } \\
\text { accessions }\end{array}$ \\
\hline Piurana & S. albornozii Correll & $a b z$ & $2,102,103,466$ & $\begin{array}{l}\text { PI 561637, GLKS } \\
\text { 35297, GLKS 35298, } \\
\text { CGN } 22731\end{array}$ & 4 & & $\begin{array}{l}\text { weak } \\
\text { evidence }\end{array}$ \\
\hline Tuberosa III & $\begin{array}{l}\text { S. achacachense } \\
\text { Cárdenas }\end{array}$ & ach & 99 & GLKS 32830 & 1 & & $\begin{array}{l}\text { not enough } \\
\text { accessions }\end{array}$ \\
\hline Tuberosa II & $\begin{array}{l}\text { S. ancophilum } \\
\text { (Correll) Ochoa }\end{array}$ & acp & 304 & CIP 761448 & 1 & $\begin{array}{l}\text { synonym of } S . \\
\text { rhomboideilanceolatum } \\
\text { Ochoa (Hawkes, 1990) }\end{array}$ & $\begin{array}{l}\text { not enough } \\
\text { accessions }\end{array}$ \\
\hline Tuberosa II & $\begin{array}{l}\text { S. acroscopicum } \\
\text { Ochoa }\end{array}$ & acs & 100 & GLKS 32436 & 1 & & $\begin{array}{l}\text { not enough } \\
\text { accessions }\end{array}$ \\
\hline Tuberosa II & S. augustii Ochoa & agu & 305 & CIP 762631 & 1 & & $\begin{array}{l}\text { not enough } \\
\text { accessions }\end{array}$ \\
\hline Tuberosa III & $\begin{array}{l}\text { S. alandiae } \\
\text { Cárdenas }\end{array}$ & aln & $\begin{array}{l}257,320,455,457 \\
458,459,460\end{array}$ & $\begin{array}{l}\text { CPC 7212, CGN } \\
\text { 18245, CGN 22349, } \\
\text { cgn962384, CGN } \\
20651, \text { CGN 18260, } \\
\text { CGN 18264 }\end{array}$ & 7 & & $\begin{array}{l}\text { weak } \\
\text { evidence for } \\
\text { combination } \\
\text { with gnd }\end{array}$ \\
\hline Tuberosa II & $\begin{array}{l}\text { S. ambosinum } \\
\text { Ochoa }\end{array}$ & $a m b$ & $104,105,467$ & $\begin{array}{l}\text { GLKS 32282, GLKS } \\
\text { 35299, CGN } 18358\end{array}$ & 3 & & no evidence \\
\hline Tuberosa II & S. amabile Vargas & $\mathrm{aml}$ & 3 & PI 365356 & 1 & $\begin{array}{l}\text { synonym of S. } \\
\text { canasense (Hawkes, } \\
\text { 1990) }\end{array}$ & $\begin{array}{l}\text { not enough } \\
\text { accessions }\end{array}$ \\
\hline Tuberosa II & $\begin{array}{l}\text { S. amayanum } \\
\text { Ochoa }\end{array}$ & amy & 302,303 & $\begin{array}{l}\text { CIP } 763004, C I P \\
763005\end{array}$ & 2 & & no evidence \\
\hline Megistacroloba & S. aracc-papa Juz. & $\operatorname{arp}$ & 109,110 & $\begin{array}{l}\text { GLKS 30082, GLKS } \\
30081\end{array}$ & 2 & $\begin{array}{l}\text { nomen dubium } \\
\text { (Hawkes, 1990) }\end{array}$ & no evidence \\
\hline Yungasensa & S. arnezii Cárdenas & arz & $\begin{array}{l}4,111,112,113 \\
471\end{array}$ & $\begin{array}{l}\text { PI 545880, GLKS } \\
\text { 32832, GLKS 32833, } \\
\text { GLKS 32834, GLKS } \\
32831\end{array}$ & 5 & & no evidence \\
\hline Megistacroloba & $\begin{array}{l}\text { S. astleyi Hawkes } \\
\text { and Hjert. }\end{array}$ & ast & $\begin{array}{l}114,472,474,475 \\
476\end{array}$ & $\begin{array}{l}\text { GLKS 32836, CGN } \\
18207, \text { CGN 18210, } \\
\text { CGN 18211, CGN } \\
18212\end{array}$ & 5 & & $\begin{array}{l}\text { weak } \\
\text { evidence for } \\
\text { combination } \\
\text { with blv } \\
\end{array}$ \\
\hline Tuberosa III & $\begin{array}{l}\text { S. avilesii Hawkes } \\
\text { and Hjert. }\end{array}$ & $\mathrm{avl}$ & $477,478,479$ & $\begin{array}{l}\text { CGN 18255, CGN } \\
18256, \text { CGN } 18257\end{array}$ & 3 & & no evidence \\
\hline Tuberosa II & $\begin{array}{l}\text { S. aymaraesense } \\
\text { Ochoa }\end{array}$ & aym & 5 & PI 607896 & 1 & & $\begin{array}{l}\text { not enough } \\
\text { accessions }\end{array}$ \\
\hline Tuberosa III & $\begin{array}{l}\text { S. vernei subsp. } \\
\text { ballsii (Hawkes) } \\
\text { Hawkes and Hjert. }\end{array}$ & bal & $906,907,908$ & $\begin{array}{l}\text { CGN 17992, CGN } \\
17993, \text { CGN } 17994\end{array}$ & 3 & & $\begin{array}{l}\text { weak } \\
\text { evidence for } \\
\text { combination } \\
\text { with vrn }\end{array}$ \\
\hline Tuberosa III & $\begin{array}{l}\text { S. berthaultii } \\
\text { Hawkes }\end{array}$ & ber & $\begin{array}{l}322,323,324,480 \\
481,482,483,484 \\
485,486,487,488 \\
489,490,491,492 \\
493,494,561^{*}, 939 \\
940,941,943,944\end{array}$ & $\begin{array}{l}\text { CGN 20644, CGN } \\
\text { 20650, CGN 18042, } \\
\text { CGN 18074, CGN } \\
\text { 18190, CGN 20635, } \\
\text { CGN 20636, CGN } \\
22715, \text { CGN18216, } \\
\text { CGN 22716, CGN } \\
20645, C G N 18246, \\
\text { CGN 23804, CGN } \\
18228, C G N 22727, \\
\text { BGRC 15479, CGN } \\
17823, \text { CGN 18118, } \\
\text { GLKS 31670*, CGN } \\
18189, \text { CGN 23508, } \\
\text { CGN 18267, CGN } \\
17716, \text { CGN } 23477\end{array}$ & 24 & & no evidence \\
\hline
\end{tabular}


Table 3 Information on species labels and accessions used in the analysis and suggestions for species status (Continued)

\begin{tabular}{|c|c|c|c|c|c|c|}
\hline Megistacroloba & S. boliviense Dunal & blv & $496,498,499$ & $\begin{array}{l}\text { CGN 18196, CGN } \\
\text { 18070, INTA } \\
73228 B\end{array}$ & 3 & $\begin{array}{l}\text { weak } \\
\text { evidence for } \\
\text { combination } \\
\text { with ast }\end{array}$ \\
\hline Tuberosa III & S. brevicaule Bitter & brc & $\begin{array}{l}327,505,506,507 \\
509,1020,1025 \\
1026,1040,1047\end{array}$ & $\begin{array}{l}\text { CGN 18231, CGN } \\
\text { 17841, CGN 18226, } \\
\text { CGN 18232, CGN } \\
\text { 22321, CGN 18030, } \\
\text { CGN 18223, } \\
\text { CGN18247, } \\
\text { CGN22322, } \\
\text { CGN22717 }\end{array}$ & 10 & no evidence \\
\hline Tuberosa II & S. bukasovii Juz. & buk & $\begin{array}{l}328,511,512,514, \\
955,971,\end{array}$ & $\begin{array}{l}\text { CGN 17683, CGN } \\
17684, \text { CGN 17737, } \\
\text { CGN 17821, CGN } \\
21305, \text { CGN } 17738\end{array}$ & 6 & no evidence \\
\hline Tuberosa II & $\begin{array}{l}\text { S. canasense } \\
\text { Hawkes }\end{array}$ & can & $\begin{array}{l}260,526,527,528 \\
529,951 \$, 952,953\end{array}$ & $\begin{array}{l}\text { CPC 2725, } \\
\text { cgn960639, CGN } \\
17722, \text { CGN 17672, } \\
\text { CGN 17589, CGN } \\
20592 \$, \text { CGN } \\
18072, \text { CGN23007 }\end{array}$ & 7 & no evidence \\
\hline Yungasensa & S. chacoense Bitter & chc & $\begin{array}{l}125,126,127,246^{*} \\
263,338,470 \$ \\
543,544,545,546 \\
547,548,549,550 \\
551\end{array}$ & $\begin{array}{l}\text { GLKS 30162, GLKS } \\
30161, \text { GLKS 30180, } \\
\text { GLKS 32343*, } \\
\text { CPC5901, CGN } \\
18248, \text { CGN 17679 } \\
\$, \text { Cgn962709, CGN } \\
18365, \text { CGN 17702, } \\
\text { CGN 22384, CGN } \\
18202, \text { CGN 18294, } \\
\text { CGN 18338, } \\
\text { cgn961764, CGN } \\
22368\end{array}$ & 15 & no evidence \\
\hline no information & $\begin{array}{l}\text { S. chillonanum } \\
\text { Ochoa }\end{array}$ & chi & 12 & PI 607890 & 1 & $\begin{array}{l}\text { not enough } \\
\text { accessions }\end{array}$ \\
\hline Tuberosa II & $\begin{array}{l}\text { S. chancayense } \\
\text { Ochoa }\end{array}$ & chn & $1,552,553$ & $\begin{array}{l}\text { VIR 20892, CGN } \\
\text { 18036, CGN } 18356 \\
\end{array}$ & 3 & no evidence \\
\hline Megistacroloba & $\begin{array}{l}\text { S. chavinense } \\
\text { Correll }\end{array}$ & chv & 11 & PI 498235 & 1 & $\begin{array}{l}\text { not enough } \\
\text { accessions }\end{array}$ \\
\hline Commersoniana & $\begin{array}{l}\text { S. commersonii } \\
\text { Dunal }\end{array}$ & $\mathrm{cmm}$ & $\begin{array}{l}265,575,576,577 \\
578,1017,1018 \\
1019,1027,1028 \\
1039,1050\end{array}$ & $\begin{array}{l}\text { CPC 5861, } \\
\text { cgn961592, } \\
\text { cgn961597, CGN } \\
18027, \text { CGN 22351, } \\
\text { CGN 17988, CGN } \\
\text { 18024, CGN 18026, } \\
\text { CGN 18327, CGN } \\
\text { 18328, GLKS 35340, } \\
\text { CGN 23492 }\end{array}$ & 12 & evidence \\
\hline Tuberosa III & $\begin{array}{l}\text { S. candolleanum P. } \\
\text { Berthault }\end{array}$ & end & $530,531,532$ & $\begin{array}{l}\text { PI 498226, CGN } \\
\text { 18132, CGN } 20603\end{array}$ & 3 & no evidence \\
\hline Tuberosa II & $\begin{array}{l}\text { S. coelestipetalum } \\
\text { Vargas }\end{array}$ & cop & $\begin{array}{l}134,135,306,307 \\
572\end{array}$ & $\begin{array}{l}\text { GLKS 35433, GLKS } \\
35434, \text { CIP 761755, } \\
\text { CIP 761999, CGN } \\
20557\end{array}$ & 5 & no evidence \\
\hline Tuberosa II & $\begin{array}{l}\text { S. } \\
\text { dolichocremastrum } \\
\text { Bitter }\end{array}$ & dcm & $147,148,149,308$ & $\begin{array}{l}\text { GLKS 32342, GLKS } \\
\text { 35348, GLKS 35349, } \\
\text { CIP 762533 }\end{array}$ & 4 & $\begin{array}{l}\text { weak } \\
\text { evidence for } \\
\text { combination } \\
\text { with chv }\end{array}$ \\
\hline Tuberosa III & S. $x$ doddsii Correll & dds & $\begin{array}{l}144,145,146,588, \\
589\end{array}$ & $\begin{array}{l}\text { GLKS 32882, GLKS } \\
32883, \text { GLKS 32880, } \\
\text { CGN 20661, CGN } \\
18359\end{array}$ & 5 & no evidence \\
\hline
\end{tabular}


Table 3 Information on species labels and accessions used in the analysis and suggestions for species status (Continued)

\begin{tabular}{|c|c|c|c|c|c|c|c|}
\hline Tuberosa III & $\begin{array}{l}\text { S. microdontum } \\
\text { subsp. } \\
\text { gigantophyllum } \\
\text { (Bitter) Hawkes and } \\
\text { hjert. }\end{array}$ & gig & $\begin{array}{l}361,362,710,711 \\
712,713,714,715 \\
956,957,960,961 \\
962,963,964,965 \\
966,967\end{array}$ & $\begin{array}{l}\text { CGN 18046, CGN } \\
18083, \text { CGN 18199, } \\
\text { CGN 20639, CGN } \\
18200, C G N 17595, \\
\text { CGN 23050, CGN } \\
21342, C G N 18295, \\
\text { CGN 23511, CGN } \\
20586, C G N 18048, \\
\text { CGN 17597, CGN } \\
18049, C G N 18084, \\
\text { CGN 18003, CGN } \\
18067, C G N 22372\end{array}$ & 18 & $\begin{array}{l}\text { synonym of S. } \\
\text { microdontum Bitter } \\
\text { (van den Berg and } \\
\text { Spooner, 1992) }\end{array}$ & $\begin{array}{l}\text { no evidence, } \\
\text { part of } \\
\text { microdontum }\end{array}$ \\
\hline Tuberosa III & $\begin{array}{l}\text { S. gandarillasii } \\
\text { Cárdenas }\end{array}$ & gnd & $\begin{array}{l}16,62,163,270 \\
346,603,\end{array}$ & $\begin{array}{l}\text { PI 597750, PI } \\
\text { 597751, GLKS } \\
\text { 32423, CPC 7044, } \\
\text { CGN 20560, CGN } \\
17590\end{array}$ & 6 & & $\begin{array}{l}\text { weak } \\
\text { evidence for } \\
\text { combination } \\
\text { with aln }\end{array}$ \\
\hline \multirow[t]{3}{*}{ Tuberosa III } & S. gourlayi Hawkes & $\mathrm{grl}$ & $\begin{array}{l}\text { 347, 604, 605, 606, } \\
607 \$, 608,609 \\
610,611,1000 \$ \\
1005,1006,1008 \\
1009,1010,1011 \\
1012,1013,1014 \\
1015,1021,1022 \\
1029,1030,1032 \\
1033,1034,1035 \\
1036 \$, 1037,1042 \\
1043 \$, 1044,1048 \\
1049,1051,1052 \$, \\
1053,1054,1055 \$\end{array}$ & 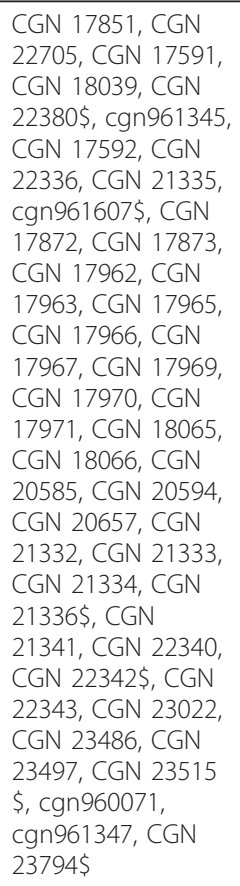 & 34 & $\begin{array}{l}\text { synonym of S. } \\
\text { leptophyes (Ochoa, } \\
\text { 1990) }\end{array}$ & no evidence \\
\hline & S. hannemanii & han & $\begin{array}{l}252^{*}, 628,629,630 \\
631,632,633\end{array}$ & $\begin{array}{l}\text { GLKS 32196*, CGN } \\
\text { 17996, CGN 17854, } \\
\text { CGN 17997, CGN } \\
20578, \text { CGN 17856, } \\
\text { CGN } 17858\end{array}$ & 7 & provisional name & $\begin{array}{l}\text { weak } \\
\text { evidence }\end{array}$ \\
\hline & S. hawkesianum & haw & $\begin{array}{l}166,167,634,635 \\
636,637,638\end{array}$ & $\begin{array}{l}\text { GLKS 32762, GLKS } \\
32765, \text { CGN 17888, } \\
\text { CGN 17889, CGN } \\
17890, \text { CGN 17891, } \\
\text { CGN } 17892\end{array}$ & 7 & provisional name & $\begin{array}{l}\text { weak } \\
\text { evidence }\end{array}$ \\
\hline Yungasensa & $\begin{array}{l}\text { S. huancabambense } \\
\text { Ochoa }\end{array}$ & hcb & $18,170,353,354$ & $\begin{array}{l}\text { PI 365359, GLKS } \\
\text { 32441, CGN 18306, } \\
\text { CGN } 17719\end{array}$ & 4 & & evidence \\
\hline Piurana & $\begin{array}{l}\text { S. hypacrarthrum } \\
\text { Bitter }\end{array}$ & hcr & 311 & CIP 761259 & 1 & & $\begin{array}{l}\text { not enough } \\
\text { accessions }\end{array}$ \\
\hline Tuberosa III & $\begin{array}{l}\text { S. hondelmannii } \\
\text { Hawkes and Hjert. }\end{array}$ & hdm & $\begin{array}{l}168,351,644,645 \\
646,647,648\end{array}$ & $\begin{array}{l}\text { GLKS 32852, CGN } \\
\text { 18106, cgn961918, } \\
\text { cgn962199, CGN } \\
\text { 18192, CGN 18193, } \\
\text { cgn962204 }\end{array}$ & 7 & & $\begin{array}{l}\text { weak } \\
\text { evidence }\end{array}$ \\
\hline
\end{tabular}


Table 3 Information on species labels and accessions used in the analysis and suggestions for species status (Continued)

\begin{tabular}{|c|c|c|c|c|c|c|}
\hline Tuberosa II & $\begin{array}{l}\text { S. humectophilum } \\
\text { Ochoa }\end{array}$ & $\mathrm{hmp}$ & 171 & GLKS 32829 & 1 & $\begin{array}{l}\text { not enough } \\
\text { accessions }\end{array}$ \\
\hline Tuberosa III & $\begin{array}{l}\text { S. hoopesii Hawkes } \\
\text { and Okada }\end{array}$ & hps & $\begin{array}{l}169,650,651,652 \\
653\end{array}$ & $\begin{array}{l}\text { GLKS 32885, CGN } \\
18363, \text { CGN 18367, } \\
\text { CGN 18368, CGN } \\
18372\end{array}$ & 5 & no evidence \\
\hline Tuberosa II & $\begin{array}{l}\text { S. huarochiriense } \\
\text { Ochoa }\end{array}$ & hro & 309 & CIP 761224 & 1 & $\begin{array}{l}\text { not enough } \\
\text { accessions }\end{array}$ \\
\hline Megistacroloba & $\begin{array}{l}\text { S. infundibuliforme } \\
\text { Phil. }\end{array}$ & ifd & $\begin{array}{l}664,665,666,667 \\
668,1007,1023\end{array}$ & $\begin{array}{l}\text { CGN 17720, CGN } \\
23063, \text { CGN 22334, } \\
\text { CGN 23048, } \\
\text { cgn960696, CGN } \\
\text { 17959, CGN } 18079\end{array}$ & 7 & no evidence \\
\hline Tuberosa II & S. immite Dunal & imt & $63,64,172$ & $\begin{array}{l}\text { PI 498245, PI } \\
\text { 365331, GLKS32819 }\end{array}$ & 3 & no evidence \\
\hline Tuberosa III & $\begin{array}{l}\text { S. incamayoense K. } \\
\text { A. Okada and A.M. } \\
\text { Clausen }\end{array}$ & inm & $\begin{array}{l}657,658,659,660 \\
661,662,663,1016\end{array}$ & $\begin{array}{l}\text { CGN 18077, CGN } \\
21320, \text { CGN 17874, } \\
\text { CGN 17875, CGN } \\
17968, \text { cgn961363, } \\
\text { CGN 22335, CGN } \\
17972\end{array}$ & 8 & no evidence \\
\hline Tuberosa III & $\begin{array}{l}\text { S. kurtzianum Bitter } \\
\text { and Wittm. }\end{array}$ & ktz & $\begin{array}{l}275,276,675,676 \\
677,678,995\end{array}$ & $\begin{array}{l}\text { CPC 5864, CPC } \\
5889, \text { CGN 22338, } \\
\text { cgn961563, CGN } \\
\text { 23042, cgn961013, } \\
\text { CGN 22353 }\end{array}$ & 7 & $\begin{array}{l}\text { weak } \\
\text { evidence }\end{array}$ \\
\hline Lignicaulia & S. lignicaule Vargas & $|g|$ & 179,685 & $\begin{array}{l}\text { GLKS 32215, CGN } \\
17723\end{array}$ & 2 & $\begin{array}{l}\text { weak } \\
\text { evidence }\end{array}$ \\
\hline Conicibaccata & $\begin{array}{l}\text { S. limbaniense } \\
\text { Ochoa }\end{array}$ & Imb & 686 & CGN 22720 & 1 & $\begin{array}{l}\text { not enough } \\
\text { accessions }\end{array}$ \\
\hline Tuberosa II & S. leptophyes Bitter & Iph & $\begin{array}{l}356,357,680,682, \\
683,684\end{array}$ & $\begin{array}{l}\text { CGN 18174, CGN } \\
18140, \text { CGN 18173, } \\
\text { CGN 18167, CGN } \\
20611, \text { CGN } 18126\end{array}$ & 6 & no evidence \\
\hline Maglia & S. maglia Schtdl. & mag & $75,76,359,688$ & $\begin{array}{l}\mathrm{PI} 245087, \mathrm{PI} \\
558316, \mathrm{CGN} \\
18064, \text { CGN } 22719 \\
\end{array}$ & 4 & no evidence \\
\hline Tuberosa III & $\begin{array}{l}\text { S. microdontum } \\
\text { Bitter }\end{array}$ & mcd & $\begin{array}{l}360,707,708,958 \\
959,994\end{array}$ & $\begin{array}{l}\text { CGN 17596, CGN } \\
22382, \text { CGN 18259, } \\
\text { CGN 20646, CGN } \\
18047, \text { CGN } 20597\end{array}$ & 6 & evidence \\
\hline Tuberosa I & $\begin{array}{l}\text { S. macropilosum } \\
\text { Correll }\end{array}$ & $m c p$ & 23,74 & $\begin{array}{l}\text { PI } 607844, \text { PI } \\
607845\end{array}$ & $\begin{array}{l}\text { synonym of S. } \\
\text { verrucosum (Spooner et } \\
\text { al. 2004) }\end{array}$ & $\begin{array}{l}\text { no evidence, } \\
\text { part of ver }\end{array}$ \\
\hline Tuberosa II & $\begin{array}{l}\text { S. mochiquense } \\
\text { Ochoa }\end{array}$ & mcq & $\begin{array}{l}186 \$, 716,717 \\
718,719\end{array}$ & $\begin{array}{l}\text { GLKS 32319\$, CGN } \\
20587, \text { CGN 18263, } \\
\text { CGN 17731, CGN } \\
21360\end{array}$ & 4 & no evidence \\
\hline Tuberosa II & S. medians Bitter & med & $\begin{array}{l}\text { 183, 691, 692, 693, } \\
694,695\end{array}$ & $\begin{array}{l}\text { GLKS 32226, CGN } \\
\text { 21349, CGN 18043, } \\
\text { CGN18308, CGN } \\
21343, \text { CGN } 18307\end{array}$ & 6 & $\begin{array}{l}\text { weak } \\
\text { evidence }\end{array}$ \\
\hline Megistacroloba & $\begin{array}{l}\text { S. megistacrolobum } \\
\text { Bitter }\end{array}$ & mga & $696,697,699,700$ & $\begin{array}{l}\text { CGN 23064, CGN } \\
\text { 17828, CGN 22347, } \\
\text { CGN 20601 }\end{array}$ & 4 & $\begin{array}{l}\text { weak } \\
\text { evidence for } \\
\text { combination } \\
\text { with tor }\end{array}$ \\
\hline Commersoniana & $\begin{array}{l}\text { S. commersonii } \\
\text { subsp. malmeanum } \\
\text { (Bitter) }\end{array}$ & $\mathrm{mlm}$ & $\begin{array}{l}139,266,579 \$ \\
580,581,1038 \\
1045,1058\end{array}$ & $\begin{array}{l}\text { GLKS 35340, CPC } \\
\text { 7520, CGN 18329\$, } \\
\text { CGN 18025, CGN } \\
\text { 18215, CGN21353, } \\
\text { CGN 22352, } \\
\text { cgn962274 }\end{array}$ & 7 & $\begin{array}{l}\text { evidence } \\
\text { (subspecies } \\
\text { or species) }\end{array}$ \\
\hline
\end{tabular}


Table 3 Information on species labels and accessions used in the analysis and suggestions for species status (Continued)

\begin{tabular}{|c|c|c|c|c|c|c|c|}
\hline Tuberosa II & $\begin{array}{l}\text { S. multidissectum } \\
\text { Hawkes }\end{array}$ & $\mathrm{mlt}$ & $\begin{array}{l}363,722,723,724, \\
725,727,728,729 \\
730,731,732\end{array}$ & $\begin{array}{l}\text { CGN 17824, CGN } \\
\text { 21344, CGN 18330, } \\
\text { cgn960739, CGN } \\
\text { 17686, CGN 17733, } \\
\text { cgn960736, CGN } \\
\text { 17825, cgn961613, } \\
\text { cgn17840, } \\
\text { cgn960967 }\end{array}$ & 11 & $\begin{array}{l}\text { synonym of } \mathrm{S} \text {. } \\
\text { bukasovii Juz. f. } \\
\text { multidissectum } \\
\text { (Hawkes) Ochoa }\end{array}$ & no evidence \\
\hline Tuberosa II & $\begin{array}{l}\text { S. marinasense } \\
\text { Vargas }\end{array}$ & $\mathrm{mrn}$ & $\begin{array}{l}77,181,182,277, \\
690\end{array}$ & $\begin{array}{l}\text { PI 607884, GLKS } \\
35430, \text { GLKS 32281, } \\
\text { CPC 7172, CGN } \\
17594\end{array}$ & 5 & & no evidence \\
\hline Tuberosa II & $\begin{array}{l}\text { S. multiinterruptum } \\
\text { Bitter }\end{array}$ & mtp & 190 & GLKS 32431 & 1 & & $\begin{array}{l}\text { not enough } \\
\text { accessions }\end{array}$ \\
\hline Tuberosa III & $\begin{array}{l}\text { S. neocardenasii } \\
\text { Hawkes and Hjert. }\end{array}$ & ncd & 193,734 & $\begin{array}{l}\text { GLKS 32855, CGN } \\
18217\end{array}$ & 2 & & no evidence \\
\hline Tuberosa III & $\begin{array}{l}\text { S. neorossii Hawkes } \\
\text { and Hjert. }\end{array}$ & nrs & $\begin{array}{l}281,735,736,737 \\
987^{*}\end{array}$ & $\begin{array}{l}\text { CPC 6047, CGN } \\
18280, C G N 17599, \\
\text { CGN 18051, CGN } \\
17763^{*}\end{array}$ & 5 & & no evidence \\
\hline Tuberosa III & $\begin{array}{l}\text { S. okadae Hawkes } \\
\text { and Hjert. }\end{array}$ & oka & $\begin{array}{l}283,365^{*}, 366,367 \\
368,739,740,741 \\
\$, 742,743,744 \\
745,746,969,970\end{array}$ & $\begin{array}{l}\text { CPC 7129, CGN } \\
18000^{*}, \text { CGN } \\
18109, \text { CGN 18108, } \\
\text { CGN 17998, CGN } \\
18269, \text { CGN 17999, } \\
\text { CGN 18279\$, } \\
\text { cgn962076, } \\
\text { cgn962078, CGN } \\
18157, \text { CGN 22709, } \\
\text { CGN 18129, CGN } \\
22703, \text { CGN } 20599\end{array}$ & 14 & & evidence \\
\hline Tuberosa III & $\begin{array}{l}\text { S. oplocense } \\
\text { Hawkes }\end{array}$ & opl & $\begin{array}{l}\text { 747, 749, 750, 751, } \\
752,753,754,1001 \\
\$, 1002,1003 \\
1004,1024,1031 \\
1041,1046,1056 \\
1057,1059\end{array}$ & $\begin{array}{l}\text { CGN 23049, } \\
\text { cgn962217, CGN } \\
21352, C G N 18088, \\
\text { CGN 18085, CGN } \\
21319, C G N 17736 \\
\$, C G N 17868, C G N \\
17869, C G N 17870, \\
\text { CGN 18087, CGN } \\
20638, C G N 22324, \\
\text { CGN 22713, CGN } \\
23798, \text { cgn961876, } \\
\text { cgn962541 }\end{array}$ & 17 & & no evidence \\
\hline Tuberosa II & S. orophilum Correll & orp & $29,83,84,196,756$ & $\begin{array}{l}\text { PI 498213, PI } \\
\text { 498209, PI 498212, } \\
\text { GLKS 35301, } \\
\text { cgn962570 }\end{array}$ & 5 & & no evidence \\
\hline Tuberosa II & $\begin{array}{l}\text { S. pampasense } \\
\text { Hawkes }\end{array}$ & pam & $288,762,763,764$ & $\begin{array}{l}\text { CPC 6024, CGN } \\
\text { 962604, CGN } \\
20575, \text { cgn960051 }\end{array}$ & 4 & & no evidence \\
\hline Tuberosa III & $\begin{array}{l}\text { S. gourlayi subsp. } \\
\text { pachytrichum } \times S \text {. } \\
\text { leptophyes }\end{array}$ & ptr & $\begin{array}{l}612,613,614,615 \\
616,617,618\end{array}$ & $\begin{array}{l}\text { cgn18102, } \\
\text { cgn18176, } \\
\text { bgrc27294, } \\
\text { bgrc27295, } \\
\text { cgn18188, } \\
\text { bgrc7231, } \\
\text { bgrc28084 }\end{array}$ & 7 & $\begin{array}{l}\text { synonym of S. } \\
\text { leptophyes (Ochoa, } \\
\text { 1990) }\end{array}$ & no evidence \\
\hline
\end{tabular}


Table 3 Information on species labels and accessions used in the analysis and suggestions for species status (Continued)

\begin{tabular}{|c|c|c|c|c|c|c|c|}
\hline Megistacroloba & $\begin{array}{l}\text { S. raphanifolium } \\
\text { Cárdenas and } \\
\text { Hawkes }\end{array}$ & rap & $\begin{array}{l}208,209,291,380 \\
790,791,792,793 \\
794,797,798,799 \\
800,801,976\end{array}$ & $\begin{array}{l}\text { GLKS 30637, GLKS } \\
\text { 30644, CPC 7090, } \\
\text { CGN 17598, } \\
\text { cgn960772, CGN } \\
\text { 20589, CGN 18300, } \\
\text { CGN 18089, } \\
\text { cgn961878, CGN } \\
18320, \text { CGN 17752, } \\
\text { CGN 18033, CGN } \\
\text { 17833, CGN 17835, } \\
\text { CGN 17822 }\end{array}$ & 15 & & evidence \\
\hline Tuberosa III & $\begin{array}{l}\text { S. xrechei Hawkes } \\
\text { and Hjert. }\end{array}$ & rch & 35 & PI 558227 & 1 & & $\begin{array}{l}\text { not enough } \\
\text { accessions }\end{array}$ \\
\hline Tuberosa III & $\begin{array}{l}\text { S. xruiz-lealii } \\
\text { Brücher }\end{array}$ & $\mathrm{rzl}$ & 802 & CGN 18117 & 1 & & $\begin{array}{l}\text { not enough } \\
\text { accessions }\end{array}$ \\
\hline Tuberosa II & $\begin{array}{l}\text { S. scabrifolium } \\
\text { Ochoa }\end{array}$ & $\mathrm{scb}$ & 37 & PI 365363 & 1 & & $\begin{array}{l}\text { not enough } \\
\text { accessions }\end{array}$ \\
\hline Tuberosa III & $\begin{array}{l}\text { S. xsucrense } \\
\text { Hawkes }\end{array}$ & scr & $\begin{array}{l}391,843,844,845 \\
846,847,848,849 \\
850\end{array}$ & $\begin{array}{l}\text { CGN 18205, CGN } \\
20628, \text { CGN 20630, } \\
\text { CGN 20631, CGN } \\
18187, \text { CGN 20634, } \\
\text { CGN 22350, CGN } \\
18206, \text { CGN } 18105\end{array}$ & 9 & & no evidence \\
\hline Megistacroloba & $\begin{array}{l}\text { S. sanctae-rosae } \\
\text { Hawkes }\end{array}$ & sct & $\begin{array}{l}803,804,805,806, \\
807,1061\end{array}$ & $\begin{array}{l}\text { CGN 20576, CGN } \\
22344, \text { CGN 17910, } \\
\text { CGN 20564, CGN } \\
17837, \text { cgn961619 } \\
\end{array}$ & 6 & & $\begin{array}{l}\text { weak } \\
\text { evidence }\end{array}$ \\
\hline Megistacroloba & $\begin{array}{l}\text { S. sogarandinum } \\
\text { Ochoa }\end{array}$ & sgr & $215,315,316,814$ & $\begin{array}{l}\text { GLKS 35382, CIP } \\
\text { 761465, CIP } \\
\text { 761586, CGN } 17601\end{array}$ & 4 & & evidence \\
\hline Tuberosa II & $\begin{array}{l}\text { S. sandemanii } \\
\text { Hawkes }\end{array}$ & snd & $93,94,808$ & $\begin{array}{l}\text { PI 607894, PI } \\
\text { 607895, CGN } 17600\end{array}$ & 3 & & $\begin{array}{l}\text { weak } \\
\text { evidence for } \\
\text { combination } \\
\text { with wbr }\end{array}$ \\
\hline Tuberosa II & S. soukupii Hawkes & sou & 815 & CGN 18061 & 1 & $\begin{array}{l}\text { synonym of } S . \\
\text { canasense (Hawkes } \\
\text { 1990) }\end{array}$ & $\begin{array}{l}\text { not enough } \\
\text { accessions }\end{array}$ \\
\hline Tuberosa III & S. spegazzinii Bitter & spg & $\begin{array}{l}217,385,386,822 \\
823,824,826,827 \\
828 \$\end{array}$ & $\begin{array}{l}\text { GLKS 32755, CGN } \\
\text { 17759, CGN 17839, } \\
\text { cgn960795, CGN } \\
21318, \text { CGN 22707, } \\
\text { CGN 21321, CGN } \\
23015, \text { CGN 18034\$ }\end{array}$ & 8 & & no evidence \\
\hline Tuberosa II & $\begin{array}{l}\text { S. sparsipilum } \\
\text { (Bitter) Juz. and } \\
\text { Bukasov }\end{array}$ & spl & $\begin{array}{l}382,383,384,816 \\
817,818,819,820 \\
821,972,973,975 \\
978,\end{array}$ & $\begin{array}{l}\text { CGN 18225, CGN } \\
18230, \text { CGN 18154, } \\
\text { CGN 18096, CGN } \\
17838, C G N 18221, \\
\text { CGN 20653, CGN } \\
17758, C G N 20602, \\
\text { CGN 18099, CGN } \\
22702, C G N 18094, \\
\text { CGN 18131 }\end{array}$ & 13 & & no evidence \\
\hline Tuberosa III & $\begin{array}{l}\text { S. xsetulosistylum } \\
\text { Bitter }\end{array}$ & stl & 214,811 & $\begin{array}{l}\text { GLKS 31014, CGN } \\
20655\end{array}$ & 2 & & no evidence \\
\hline
\end{tabular}


Table 3 Information on species labels and accessions used in the analysis and suggestions for species status (Continued)

\begin{tabular}{|c|c|c|c|c|c|c|}
\hline Tuberosa III & S. tarijense Hawkes & $\operatorname{tar}$ & $\begin{array}{l}224,225,280^{*}, 392, \\
852,853,854,855, \\
856,857,858,859, \\
860,862,863,864, \\
865,866,867,868, \\
869,870,871,872 \\
873,874,875,876, \\
877,878,879\end{array}$ & $\begin{array}{l}\text { GLKS 31570, GLKS } \\
\text { 31572, CPC 7208*, } \\
\text { CGN 17861, CGN } \\
\text { 22729, cgn962224, } \\
\text { CGN 22714, CGN } \\
\text { 18198, cgn960807, } \\
\text { cgn960805, } \\
\text { cgn960806, CGN } \\
\text { 17975, cgn961432, } \\
\text { CGN 21337, CGN } \\
\text { 23795, cgn961736, } \\
\text { CGN 17976, CGN } \\
\text { 17974, CGN 17977, } \\
\text { CGN 18107, } \\
\text { cgn961128, CGN } \\
17978, \text { CGN 17979, } \\
\text { cgn961441, CGN } \\
17980, \text { CGN 21338, } \\
\text { CGN 17981, } \\
\text { cgn961449, CGN } \\
17982, \text { cgn961451, } \\
\text { cgn962690 }\end{array}$ & 31 & no evidence \\
\hline Megistacroloba & $\begin{array}{l}\text { S. megistacrolobum } \\
\text { subsp. } \\
\text { toralapanum } \\
\text { (Cárdenas and } \\
\text { Hawkes) }\end{array}$ & tor & $\begin{array}{l}278,701,702,703, \\
704,705,706\end{array}$ & $\begin{array}{l}\text { CPC 1773, CGN } \\
17728, \text { CGN 23006, } \\
\text { CGN 18145, CGN } \\
18146, \text { CGN 18147, } \\
\text { CGN } 18125\end{array}$ & 7 & $\begin{array}{l}\text { weak } \\
\text { evidence for } \\
\text { combination } \\
\text { with mga }\end{array}$ \\
\hline Tuberosa III & $\begin{array}{l}\text { S. ugentii Hawkes } \\
\text { and K. A. Okada }\end{array}$ & ugt & $44,248,249,892$ & $\begin{array}{l}\text { PI 546029, GLKS } \\
\text { 32887, GLKS 32889, } \\
\text { CGN 18369 }\end{array}$ & 4 & no evidence \\
\hline Tuberosa I & $\begin{array}{l}\text { S. verrucosum } \\
\text { Schtdl. }\end{array}$ & ver & $\begin{array}{l}393,825^{*}, 909,910 \\
911,912,914,915 \\
916,917,918,919 \\
920,921,922,923 \\
988,989,990\end{array}$ & $\begin{array}{l}\text { CGN 17768, CGN } \\
18100 * \text {, CGN } \\
22326, \text { CGN 22374, } \\
\text { CGN 17764, CGN } \\
20567, \text { CGN 17769, } \\
\text { CGN 17765, CGN } \\
17773, \text { CGN 17771, } \\
\text { CGN 17766, CGN } \\
17770, \text { CGN 17772, } \\
\text { cgn960832, } \\
\text { cgn960833, CGN } \\
20566, \text { CGN 23017, } \\
\text { CGN 17767, CGN } \\
17774\end{array}$ & 19 & evidence \\
\hline Tuberosa III & $\begin{array}{l}\text { S. gourlayi subsp. } \\
\text { vidaurrei (Cárdenas) } \\
\text { Hawkes and hjert. }\end{array}$ & vid & $\begin{array}{l}619,620,621,622 \\
623,624,625,626\end{array}$ & $\begin{array}{l}\text { CGN 17848, CGN } \\
17849, \text { CGN 18040, } \\
\text { CGN 17850, CGN } \\
18038, \text { CGN 17864, } \\
\text { CGN 23024, CGN } \\
23045\end{array}$ & 8 & no evidence \\
\hline Conicibaccata & $\begin{array}{l}\text { S. } \\
\text { violaceimarmoratum } \\
\text { Bitter }\end{array}$ & vio & $924,925,926$ & $\begin{array}{l}\text { CGN 18296, CGN } \\
20647, \text { CGN } 22878\end{array}$ & 3 & no evidence \\
\hline Tuberosa II & S. velardei Ochoa & $\mathrm{vlr}$ & 97,893 & $\begin{array}{l}\text { Pl 619114, CGN } \\
18324\end{array}$ & 2 & no evidence \\
\hline Tuberosa III & $\begin{array}{l}\text { S. venturii Hawkes } \\
\text { and Hjert. }\end{array}$ & vnt & $250,894,896,993$ & $\begin{array}{l}\text { GLKS 32794, CGN } \\
17761, \text { cgn961508, } \\
\text { CGN } 17755\end{array}$ & 4 & $\begin{array}{l}\text { weak } \\
\text { evidence }\end{array}$ \\
\hline Tuberosa III & $\begin{array}{l}\text { S. virgultorum } \\
\text { (Bitter) Cárdenas } \\
\text { and Hawkes }\end{array}$ & $\operatorname{vrg}$ & $\begin{array}{l}927,928,929,930 \\
931,932 \$\end{array}$ & $\begin{array}{l}\text { cgn962448, CGN } \\
17775, \text { cgn962072, } \\
\text { CGN 20615, } \\
\text { cgn962077, CGN } \\
20652 \$\end{array}$ & 5 & no evidence \\
\hline
\end{tabular}


Table 3 Information on species labels and accessions used in the analysis and suggestions for species status (Continued)

\begin{tabular}{|c|c|c|c|c|c|c|}
\hline Tuberosa III & $\begin{array}{l}\text { S. vernei Bitter and } \\
\text { Wittm. }\end{array}$ & vrn & $\begin{array}{l}895^{*}, 897,898,899, \\
900,901,902,903, \\
904,905 \$, 979, \\
980,981,982,983 \\
984,985,986\end{array}$ & $\begin{array}{l}\text { CGN 17762*, CGN } \\
22728, C G N 18111, \\
\text { CGN 21350, CGN } \\
22345, C G N 18112, \\
\text { CGN 18114, CGN } \\
23039, C G N 18278, \\
\text { CGN 17836\$, CGN } \\
18110, C G N 21315, \\
\text { CGN 17995, CGN } \\
18113, C G N 18115 \\
\text { CGN 23516, CGN } \\
18277, \text { cgn963094 }\end{array}$ & 17 & $\begin{array}{l}\text { weak } \\
\text { evidence for } \\
\text { combination } \\
\text { with bal }\end{array}$ \\
\hline Tuberosa II & S. weberbaueri & wbr & 254,300 & $\begin{array}{l}\text { GLKS 32725, CPC } \\
6032\end{array}$ & 2 & $\begin{array}{l}\text { weak } \\
\text { evidence for } \\
\text { combination } \\
\text { with snd }\end{array}$ \\
\hline Yungasensa & $\begin{array}{l}\text { S. yungasense } \\
\text { Hawkes }\end{array}$ & yun & $98,934 \$, 935,936$ & $\begin{array}{l}\mathrm{PI} 614703, \text { CGN } \\
18336 \$, \text { CGN } \\
20677, \text { CGN } 20676\end{array}$ & 3 & no evidence \\
\hline
\end{tabular}

(personal communication R. Hoekstra, CGN). The accessions labeled S. microdontum, S. huancabambense and S. sogarandinum share their cluster with accessions from other species, but the optimal partitioning of genetic variation within the cluster shows that they represent distinct genetic units. This is consistent with the results from Jacobs et al. [9] and most of these species were also recognized in one or more other studies $[2,6,32,36,37]$.

\section{Support for combinations of species, pointing at overclassification}

Some species are assigned to one STRUCTURE cluster, but their accessions do not form distinct genetic units within the cluster on their own, but combined with accessions from another species they do (Table 2). These are probably cases of overclassification. Examples are the combination of S. verrucosum and S. macropilosum in cluster 2, of S. kurtzianum and S. maglia in cluster 3, of S. venturii and S. okadae in cluster 3, of S. sandemanii, $S$. weberbauerii, and $S$. medians in cluster 5 . Some of these combinations have already been recognized in the literature, e.g. S. macropilosum is considered a synonym of $S$. verrucosum [6].

Spooner and Salas [2] recognized S. medians and $S$. sandemanii, but not $S$. weberbauerii, which name they apparently considered as a synonym (unfortunately, information about this was not provided). Spooner et al. [38] synonymized both S. sandemanii and S. weberbaueri under $S$. medians.

\section{Accessions scattered across clusters, pointing at mislabelling}

The analysis showed that accessions from some species were scattered across two or even three clusters. This was the case for the accessions with the following species labels: S. maglia, S. doddsii, S. chacoense, S. gourlayi, S. virgultorum, S. hoopesii, S. augustii, S. tarijense S. vernei, S. infundibuliforme, S. alandiae, S. neorosii, S. sucrense, S. pachytrichum, and S. violaceimarmoratum. A major cause for this situation is probably mislabeling of accessions, although some of these species may be the product of hybridization events that occurred a long time ago. For instance, Solanum doddsii from Bolivia has been hypothesized to be a hybrid between $S$. alandiae and S. chacoense [39].

Misclassifications do occur since identification is often problematic due to ambiguous species characteristics. Problems with the identification of species were already addressed by Spooner and Salas [2] and Spooner and van den Berg [40], who noted that many of the taxa are extremely similar in morphology and many species are distinguished only by minor characters with often overlapping character states.

\section{Hybrid accessions}

Many authors $[1,2,4,41,42]$ have suggested that certain recognized species in Solanum sect. Petota are the results of hybridization. Recent hybridizations can readily be recognized from the STRUCTURE analysis by the probability with which they are assigned to a particular cluster. While most accessions have a very high probability (usually around 0.9) to belong to one cluster, hybrid individuals tend to have a much lower probability $(<0.5)$ and have a, often only slightly lower, probability to belong to another cluster. Schulte et al. [43] also argue that a posterior probability lower than 0.5 provides strong evidence for a recent hybrid origin of individuals.

To practically present our results, we have assigned all accessions to the cluster to which it had the highest 
probability, but Additional file 1 lists all probabilities for all accessions. Hybrid accessions thus identified include amongst others accessions of the species $S$. spegazzinii and S. gourlayi, which co-occur in northern Argentina. The S. spegazzinii accession SPG386 was assigned to cluster 3 with a probability of 0.459 and with 0.262 to cluster 16. Another example of recent hybridization is NRS737 which shows probabilities of 0.435 and 0.434 with the clusters 13 and 15, respectively. However, in all cases the actual parents are unknown.

\section{Non-supported species}

Some species do appear in one cluster in the STRUCTURE analysis, but their accessions do not form a separate group in the Fst analysis, not even as part of a fixed combination with another species label. This concerns the following species: S. mochiquense, S. immite, S. chancayense in cluster 7, S. canasense, S. bukasovii, S. candolleanum, S. coelestipetalum, S. pampasense, S. ambosinum, S. marinasense, S. multidissectum, S. velardei in cluster 10, S. arnezii, S. yungasense, in cluster 12, S. incamayoense in cluster 13, S. tarijense, S. berthaultii in cluster 14, S. arac-pappa, S. leptophyes, S. ugentii, S. oplocense, S. sparsipilum, and S. brevicaule in cluster 16 .

Many species mentioned in this category are part of what is termed the 'brevicaule complex' $[7,8,44]$ : $S$. canasense S. bukasovii, S. candolleanum, S. coelestipetalum, S. pampasense, S. ambosinum, S. marinasense, S. velardei, S. incamayoense, S. leptophyes, S. ugentii and S. sparsipilum. Ugent [45] already proposed in 1970 that these should be reduced to one species. The division of the species according to our analysis in two clusters (10 and 16) reflects the presence of the northern and southern subgroups of the brevicaule-complex (see below). Solanum oplocense was shown to be a welldefined species using morphological data [7] and molecular data [8], but it was not distinct in an AFLP study [46] nor in ours. Previous results from a morphological study [47] and a more recent molecular study [48] had already suggested that the species $S$. berthaultii and $S$. tarijense should be combined. The species in cluster 7 were studied morphologically by Ames and collaborators [49], who placed Solanum immite and S. chancayense among the 6 distinctive species in a group of 29 species, the remainder of which were 'difficult to distinguish'.

\section{Clusters correspond to the geographical origin of the accessions}

Many accessions within a cluster come from the same geographical region (Additional file 1). For the largest and most complicated clusters $(7,10,12,14,16)$ the information on the geographic origin of the accessions allows to draw some tentative conclusions. Cluster 16 contains mostly accessions from Argentina and Bolivia from the southern brevicaule complex and cluster 10 consist mostly of accessions from Peru (and northern Bolivia) that can be considered as belonging to the northern brevicaule complex. This separation of the brevicaule complex in a northern and southern part was already noted by Kardolus [50], was confirmed by Spooner and Salas [2] and is accepted in the treatment of this group on the Solanaceae Source website (http:// www.solanaceaesource.org), where Spooner and his collaborators maintain two species, S. candolleanum for the northern representatives, and $S$. brevicaule for the southern representatives. Cluster 7 contains almost exclusively Peruvian accessions, and some species labels in cluster 7 (S. albornozii, S. augustii, S. chancayense, S. dolichocremastrum, S. immite) are associated with series Piurana [1,2], but Jacobs et al. [9] could not find support for these species to be included in one of the recognized Piurana species groups. Ames and collaborators $[49,51]$ studied putative members of series Piurana with, respectively, morphological data and COSII markers, and concluded that based on morphology only three out of a total of 33 species could be recognized. The molecular data supported more species, some of them lacking morphological support, and the authors announced that decisions on species boundaries will be formalized in a forthcoming taxonomic monograph.

Cluster 14 contains all S. berthaultii accessions and almost all $S$. tarijense accessions, plus a few accessions with other species labels, which mostly come from Bolivia and Argentina. Cluster 12 contains accessions from various geographical origins, most of them from Bolivia and Argentina but some are from Peru and Paraguay. This group may represent accessions that relatively easily exchanged genetic material. The geographical distribution of accessions within clusters is consistent with the notion that our approach produces a meaningful arrangement of the accessions into groups that may (have) exchange(d) genetic material. For exchange of genetic material at least the accessions with the different species labels should have overlapping or adjacent geographical areas, at present or in the recent past.

Indeed, information on the distribution areas of the species of sect. Petota given in Hijmans et al. [52] confirms overlapping areas for many species within the recognized clusters, e.g. the species $S$. augustii, S. immite and S. dolichocremastrum in cluster 7, and S. berthaultii and S. tarijense in cluster 14.

\section{Conclusion}

A large number of species is presently recognized in the group of South American representatives of Solanum section Petota. The approach taken in the present paper was to determine the genetic distinctiveness of these 
species. The outcome questions the species and subspecies status of more than half of the taxonomic labels used in South American part of Solanum section Petota. The genetically distinct clusters and groups within clusters resulting from our analysis can be used as a basis for recognizing groups of species and for an evaluation of species status (Table 3).

\section{Additional material}

Additional file 1: Plant material used and cluster assignment. This file contains information on the accession numbers and geographic origin of the 566 samples used in this study. Also indicated is the cluster to which an accession has been assigned. The table lists all probabilities for all accessions. In this file putative hybrid accessions may readily be detected through conditional formatting (probabilities above 0.5 are in dark grey cells, lower probabilities - that may be indicative of recent hybridisation - in white cells, and negligible probabilities in light grey font).

\section{Acknowledgements}

This project was financed by the Centre of Biosystems Genomics (CBSG) which is part of the Netherlands Genomics Initiative/Netherlands Organization for Scientific Research.

The acronym AFLP is a registered trademark (AFLP ${ }^{\circledR}$ ) of Keygene N.V. and the AFLP ${ }^{\circledR}$ technology is covered by patents and patent applications of KeyGene N.V.

\section{Author details}

${ }^{1}$ Wageningen UR Plant Breeding, Wageningen University and Research Centre, Droevendaalsesteeg 1, 6708PB Wageningen, The Netherlands. ${ }^{2}$ Biosystematics, Wageningen University and Research Centre, Wageningen, The Netherlands. ${ }^{3}$ Centre for BioSystems Genomics, P.O. Box 98, 6700 AB Wageningen, The Netherlands.

\section{Authors' contributions}

MJ carried out the analyses and drafted the manuscript, RVB, MS and BV participated in coordination and design of the study and in writing the manuscript. All authors read and approved of the final manuscript.

Received: 30 June 2010 Accepted: 10 February 2011

Published: 10 February 2011

\section{References}

1. Hawkes JG: The potato, Evolution, Biodiversity and Genetic Resources. London. Belhaven Press; 1990.

2. Spooner DM, Salas A: Structure, Biosystematics, and genetic resources. In Handbook of potato production, improvement, and post-harvest management. Edited by: Gopal J, Khurana SMP. New York: The Haworth Press; 2006:1-39.

3. van den Berg RG, Jacobs MMJ: Molecular Taxonomy. In Potato Biology and Biotechnology. Edited by: Vreugdehil D. Oxford: Elsevier; 2007:55-76.

4. Correll DS: The potato and its wild relatives. Renner: Texas Research Foundation; 1962.

5. Spooner DM, Hijmans RJ: Potato systematics and germplasm collecting, 1989-2000. Am J Potato Res 2001, 78:237-278.

6. Spooner DM, van den Berg RG, Rodríguez A, Bamberg J, Hijmans RJ, LaraCabrera SI: Wild Potatoes (Solanum section Petota; Solanaceae) of North and Central America. In Systematic Botany Monographs. Edited by: McPherson GD, Prather LA, Ranker TA, Reznicek AA. USA: The American Society of Plant Taxonomists; 2004

7. van den Berg RG, Miller JT, Ugarte ML, Kardolus JP, Villand J, Nienhuis J, Spooner DM: Collapse of morphological species in the wild potato Solanum brevicaule complex (Solanaceae: sect. Petota). Am J Bot 1998, 85:92-109.
8. Miller JT, Spooner DM: Collapse of species boundaries in the wild potato Solanum brevicaule complex (Solanaceae, Solanum sect. Petota): molecular data. Plant Syst Evol 1999, 214:103-130.

9. Jacobs MMJ, van den Berg RG, Vleeshouwers VGAA, Visser M, Mank R, Sengers M, Hoekstra R, Vosman B: AFLP analysis reveals a lack of phylogenetic structure within Solanum section Petota. BMC Evol Biol 2008, 8:1-12.

10. Falush D, Stephens M, Pritchard JK: Inference of Population Structure Using Multilocus Genotype Data: Linked Loci and Correlated Allele Frequencies. Genetics 2003, 164:1567-1587.

11. Pritchard JK, Stephens M, Donnelly P: Inference of Population Structure Using Multilocus Genotype Data. Genetics 2000, 155:945-959.

12. Rosenberg NA, Pritchard JK, Weber JL, et al: Genetic structure of human populations. Science 2002, 298:2381-2385.

13. Pineiro R, Aguilar JF, Munt DD, Feliner GN: Ecology matters: AtlanticMediterranean disjunction in the sand-dune shrub Armeria pungens (Plumbaginaceae). Mol Ecol 2007, 16:2155-2171.

14. Rosenberg NA, Burke T, Elo K, et al: Empirical Evaluation of Genetic Clustering Methods Using Multilocus Genotypes From 20 Chicken Breeds. Genetics 2001, 159:699-713.

15. Coart E, Vekemans X, Smulders MJM, Wagner I, van Huylenbroeck J, van Bockstaele E, Roldán-Ruiz I: Genetic variation in the endangered Wild apple (Malus sylvestris (L.) Mill.) in Belgium as revealed by AFLP and microsatellite markers. Consequences for conservation. Mol Ecol 2003, 12:845-857.

16. Koopman WJM, Li Y, Coart E, van de Weg WE, Vosman B, Roldán-Ruiz I, Smulders MJM: Linked versus unlinked markers: multilocus microsatellite haplotype sharing as a tool to estimate gene flow and introgression. Mol Ecol 2007, 16:243-256.

17. Schenk MF, Thienpont C, Koopman WJM, Gilissen LJWJ, Smulders MJM: Phylogenetic relationships in Betula (Betulaceae) based on AFLP markers. Tree Genet Genom 2008, 4:911-924.

18. Shaffer HB, Thomson RC: Delimiting species in recent radiations. Syst Biol 2007, 56:896-906.

19. McCormack JE, Peterson AT, Bonaccorso E, Smith TB: Speciation in the highlands of Mexico: genetic and phenotypic divergence in the Mexican jay (Aphelocoma ultramarina). Mol Ecol 2008, 17:2505-2521.

20. Wolf JBW, Tautz D, Trillmich F: Galápagos and Californian sea lions are separate species: Genetic analysis of the genus Zalophus and its implications for conservation management. Front Zool 2007, 4:20.

21. Mayden RL: A hierarchy of species concepts: The denouement in the saga of the species problem. In Species. Edited by: Clardge MF, Dawah HA, Wilson MR. Chapman and Hall, London; 1997:381-424.

22. De Queiroz K: The general lineage concept of species. In Endless forms: Species and Speciation. Edited by: Howard J, Berlocher SH. Oxford University Press, New York; 1998:57-75.

23. De Queiroz K: Ernst Mayr and the modern concept of species. Proc Natl Ac Sci USA 2005, 102:6600-6607.

24. Vos P, Hogers R, Bleeker M, et al: AFLP: a new technique for DNA fingerprinting. Nucl Acids Res 1995, 23:4407-4414.

25. Rosenberg NA: Distruct: A program for the graphical display of population structure. Mol Ecol Notes 2004, 4:137-138.

26. Falush D, Stephens $M$, Pritchard JK: Inference of population structure using multilocus genotype data: dominant markers and null alleles. $\mathrm{Mol}$ Ecol Notes 2007, 7:574-578.

27. Evanno G, Regnaut S, Goudet J: Detecting the number of clusters of individuals using the software structure: a simulation study. Mol Ecol 2005, 14:2611-2620.

28. Corander J, Marttinen P, Siren J, Tang J: Enhanced Bayesian modelling in BAPS software for learning genetic structures of populations. BMC Bioinformatics 2008, 9:539.

29. Jing R, Vershinin A, Grzebyta J, Shaw P, Smýkal P, Marshall D, Ambrose MJ, Ellis TH, Flavell AJ: The genetic diversity and evolution of field pea (Pisum) studied by high throughput retrotransposon based insertion polymorphism (RBIP) marker analysis. BMC Evol Biol 2010, 10:44.

30. Vekemans $X$, Beauwens T, Lemaire M, Roldán-Ruiz I: Data from amplified fragment length polymorphism (AFLP) markers show indication of size homoplasy and of a relationship between degree of homoplasy and fragment size. Mol Ecol 2002, 11:139-151.

31. Zhivotovsky LA: Estimating population structure in diploids with multilocus dominant DNA markers. Mol Ecol 1999, 8:907-913. 
32. van den Berg RG, Spooner DM: A reexamination of infraspecific taxa of a wild potato, Solanum microdontum (Solanum sect. Petota: Solanaceae). Plant Syst Evol 1992, 182:239-252.

33. Giannattasio RB, Spooner DM: A reexamination of species boundaries between Solanum megistacrolobum and S. toralapanum (Solanum sect. Petota, series Megistacroloba): morphological data. Syst Bot 1994, 19:89-105.

34. Giannattasio RB, Spooner DM: A reexamination of species boundaries and hypotheses of hybridization concerning Solanum megistacrolobum and S. toralapanum (Solanum sect. Petota, series Megistacroloba): Molecular data. Syst Bot 1994, 19:106-115.

35. Spooner DM, Ugarte ML, Skroch PW: Species boundaries and interrelationships of two closely related sympatric diploid wild potato species, Solanum astleyi and S. boliviense, based on RAPDs. Theor Appl Genet 1997, 95:764-771.

36. Spooner DM, Sytsma KJ, Smith JF: A molecular reexamination of diploid hybrid speciation of Solanum raphanifolium. Evolution 1991, 45:757-764.

37. Castillo RO, Spooner DM: Phylogenetic Relationships of Wild Potatoes, Solanum Series Conicibaccata (Sect. Petota). Syst Bot 1997, 22:45-83.

38. Spooner DM, Fajardo D, Salas A: Revision of the Solanum medians complex (Solanum section Petota). Syst Bot 2008, 33:579-588.

39. Hawkes JG, Hjerting JP: The potatoes of Bolivia: their breeding value and evolutionary relationships. Oxford University Press, Oxford, UK; 1989.

40. Spooner DM, van den Berg RG: An analysis of recent taxonomic concepts in wild potatoes (Solanum sect. Petota). Genet Res Crop Evol 1992, 39:23-37.

41. Masuelli RW, Camadro EL, Erazzú LE, Bedogni MC, Marfil CF: Homoploid hybridization in the origin and evolution of wild diploid potato species. Plant Syst Evol 2009, 277:143-151.

42. Erazzú LE, Camadro EL, Clausen AM: Persistence over time, overlapping distribution and molecular indications of interspecific hybridization in wild potato populations of Northwest Argentina. Euphytica 2009, 168:249-262

43. Schulte K, Silvestro D, Kiehlmann E, Vesely S, Novoa P, Zizka G: Detection of recent hybridization between sympatric Chilean Puya species (Bromeliaceae) using AFLP markers and reconstruction of complex relationships. Mol Phylogenet Evol 2010, 57:1105-1119.

44. Alvarez NMB, Peralta IE, Salas A, Spooner DM: A morphological study of species boundaries of the wild potato Solanum brevicaule complex: replicated field trials in Peru. Plant Syst Evol 2008, 274:37-45.

45. Ugent D: The Potato. Science 1970, 170:1161-1166.

46. Spooner DM, McLean K, Ramsay G, Waugh R, Bryan GJ: A single domestication for potato based on multilocus amplified fragment length polymorphism genotyping. Proc Natl Acad Sci USA 2005, 102:14694-16499.

47. Spooner DM, van den Berg RG: Species limits and hypotheses of hybridization of Solanum berthaultii Hawkes and S. tarijense Hawkes: morphological data. Taxon 1992, 41:685-700.

48. Spooner DM, Fajardo D, Bryan GJ: Species limits of Solanum berthaultii Hawkes and S. tarijense Hawkes and the implications for species boundaries in Solanum sect. Petota. Taxon 2007, 56:987-999.

49. Ames M, Salas A, Spooner DM: A morphometric study of species boundaries of the wild potato Solanum series Piurana (Solanaceae) and putatively related species from seven other series in Solanum sect. Petota. Syst Bot 2008, 33:566-578.

50. Kardolus JP: A biosystematic analysis of Solanum acaule. PhD thesis Wageningen Agricultural University; 1998.

51. Ames M, Spooner DM: Phylogeny of Solanum series Piurana and related species in Solanum section Petota based on five conserved ortholog sequences. Taxon 2010, 59:1091-1101.

52. Hijmans RJ, Spooner DM: Geographic distribution of wild potato species. Am J Bot 2001, 88:2101-2112.

doi:10.1186/1471-2148-11-42

Cite this article as: Jacobs et al:: What's in a name; Genetic structure in Solanum section Petota studied using population-genetic tools. BMC Evolutionary Biology 2011 11:42.

\section{Submit your next manuscript to BioMed Central and take full advantage of:}

- Convenient online submission

- Thorough peer review

- No space constraints or color figure charges

- Immediate publication on acceptance

- Inclusion in PubMed, CAS, Scopus and Google Scholar

- Research which is freely available for redistribution

Submit your manuscript at www.biomedcentral.com/submit
C Biomed Central 